\title{
Pacific
}

Journal of

Mathematics

\section{INVARIANT FINSLER METRICS ON POLAR HOMOGENEOUS SPACES}

ShaOQIANG DENG

Volume $247 \quad$ No. 1 


\title{
INVARIANT FINSLER METRICS ON POLAR HOMOGENEOUS SPACES
}

\author{
SHAOQIANG DENG
}

\begin{abstract}
We study invariant Finsler metrics on polar homogeneous manifolds. After establishing existence results, we prove that an invariant Finsler metric on a nonsymmetric polar homogeneous manifold of a simply connected compact simple Lie group is Berwaldian if and only if it is Riemannian. As an application, we prove that on each such manifold with generalized rank of at least 2, there exist infinitely many invariant Finsler metrics that are reversible, non-Berwaldian and of vanishing $S$-curvature; this kind of space is sought after in an open problem of Shen. Finally, using one type of polar homogeneous manifold, we give a classification of homogeneous Randers spaces with positive constant flag curvature.
\end{abstract}

\section{Introduction}

A fundamental problem in Riemann-Finsler geometry is that of classifying the Finsler metrics on a given manifold. In full generality, this problem is intractable, so we must focus on metrics with certain special properties (particularly curvature properties), such as spaces of constant flag curvature and spaces with isotropic S-curvature. One of the most important advances has been the classification of Randers metrics with constant flag curvature obtained by Bao et al. [2004]. Also important is the work of Szabó [1981; 2006] on symmetric Berwald spaces.

Here we consider this problem for invariant Finsler metrics on homogeneous manifolds. More precisely, let $G$ be a Lie group and $H$ be a closed subgroup of $G$. Then the coset space $G / H$ admits a (unique) differentiable structure such that the action of $G$ on $G / H$ is smooth; that is, $G$ can be viewed as a Lie transformation group on the manifold $G / H$. Our goal is to classify the $G$-invariant Finsler metrics on $G / H$ and study the geometrical properties of such metrics. In previous work, we have obtained some partial results. For example, in [Deng and Hou 2004a], we proved that there exist invariant non-Riemannian Finsler metrics on $G / H$, provided

MSC2000: 22E46, 53C12, 53C60.

Keywords: polar actions, Minkowski representations, generalized rank, Berwald spaces, Randers metrics.

Supported by NSFC of China (grant 10971104). 
$H$ is compact and the action of $H$ on the tangent space of $G / H$ at the origin $o=e H$ (that is, the action of the linear isotropic representation) is not irreducible; in other words, we assumed that there exist nontrivial invariant subspaces of $H$. However, when the linear isotropic representation is irreducible, the situation is very complicated. For example, if $(G, H)$ is a Riemannian symmetric pair and the symmetric space $G / H$ is irreducible of rank 1 , then any $G$-invariant Finsler metric on $G / H$ must be Riemannian. However, if the rank is at least 2, then there exist infinitely many $G$-invariant Finsler metrics on $G / H$ that are non-Riemannian [Szabó 1981]. Therefore it is interesting to find the conditions under which a coset space with irreducible linear isotropic representation has invariant non-Riemannian Finsler metrics and to classify those metrics.

Coset spaces with irreducible linear isotropic representation are called isotropic irreducible homogeneous spaces. Wolf [1968; 1977] has studied the interesting geometry of these manifolds extensively. It is known that a connected, simply connected, noncompact, isotropic irreducible homogeneous space is either flat or a Riemannian symmetric space [Besse 1987]. Therefore we are only interested in the compact case. In this case, a classification of a special type of such spaces (strongly isotropic irreducible homogeneous spaces) was obtained independently by Manturov [1961a; 1961b; 1966], Wolf [1968], and Krámer [1975]. Wang and Ziller [1991] studied a more generalized class of Riemannian spaces: isotropy irreducible Riemannian spaces. It turns out that many such spaces are nonsymmetric. So, our first step is to classify the invariant Finsler metrics on compact isotropic irreducible homogeneous manifolds.

A deep analysis of this problem shows that a general classification is unreachable even if we confine ourselves to the isotropic irreducible homogeneous spaces. However, the situation simplifies if the isotropic representation is polar, meaning that there exists a subspace of the tangent space that intersects every orbit of the isotropic group and does so perpendicularly at any intersection. Then the algebraic methods of representation theory are available, and we can obtain a satisfactory classification theorem.

Our main results can be summarized as follows: We first use the notion of Minkowski representations of Lie groups to refine Szabó's result on the existence of invariant non-Riemannian Finsler metrics on Riemannian symmetric spaces to establish a bijection between the invariant Finsler metrics on a polar homogeneous space and the Weyl-invariant Minkowski norms on a generalized Cartan space. In the compact case, we study the geometric properties of such metrics. In particular, we prove that an invariant Finsler metric on a nonsymmetric polar homogeneous manifold of a simply connected compact simple Lie group is Berwaldian if and only if it is Riemannian. As an application, we prove that on each nonsymmetric polar homogeneous manifold of a compact simple Lie group with generalized rank 
of at least 2, there exist infinitely many invariant Finsler metrics that are reversible, non-Berwaldian and have vanishing S-curvature. Finally, using one type of polar homogeneous manifold, we obtain a classification of homogeneous Randers spaces with positive constant flag curvature. The bijection established here is new, and can be viewed as a way to classify invariant Finsler metrics on polar homogeneous spaces. We also mention that Szabó gave a classification of invariant Berwald metrics on Riemannian symmetric spaces by explicit construction via Chevalley polynomials. It is an interesting problem to consider the generalization of his method to the polar cases.

This paper is organized as follows. In Sections 1 and 2, we recall the general properties of Minkowski representations and polar representations. Section 3 gives our classification of invariant Finsler metrics on polar homogeneous manifolds, while Section 4 classifies invariant Finsler metrics in general. In Section 5, we study the geometrical properties of such metrics. In Section 6, we give a complete classification of invariant Randers metrics on polar homogeneous manifolds.

\section{Minkowski representations of Lie groups}

Definition 1.1. Let $V$ be an $n$-dimensional real vector space. A Minkowski norm on $V$ is a functional $F$ on $V$ that is smooth on $V \backslash\{0\}$ and satisfies these conditions:

- $F(u) \geq 0$ for all $u \in V$.

- $F(\lambda u)=\lambda F(u)$ for all $\lambda>0$.

- For any basis $\varepsilon_{1}, \varepsilon_{2}, \ldots, \varepsilon_{n}$ of $V$, write $F(y)=F\left(y^{1}, y^{2}, \ldots, y^{n}\right)$, where $y=y^{j} \varepsilon_{j}$. Then the Hessian matrix

$$
\left(g_{i j}\right):=\left(\left[\frac{1}{2} F^{2}\right]_{y^{i} y^{j}}\right)
$$

is positive definite at any point of $V \backslash\{0\}$.

If $V$ is a real vector space endowed with a Minkowski norm $F$, then $(V, F)$ is called a Minkowski space. Minkowski spaces play a role in Finsler geometry analogous to the role Euclidean spaces play in Riemannian geometry. In fact, a Finsler space is just a smooth manifold endowed with a smoothly varying family of Minkowski norms on its tangent spaces. Unlike in Riemannian manifolds, in a Finsler space the Minkowski norms in different tangent spaces may not be linearly isomorphic to each other.

Definition 1.2. Let $G$ be a Lie group, and $(V, \rho)$ a (real) representation of $G$. If $F$ is a Minkowski norm on $V$ such that

$$
F(\rho(g) v)=F(v) \quad \text { for all } g \in G \text { and } v \in V,
$$

then we call $(V, \rho, F)$ a Minkowski representation of $G$. 
The notion of Minkowski representations of Lie groups is a natural and obvious generalization of orthogonal representations.

Proposition 1.3 [Deng and Hou 2004a]. Suppose G/H is a coset space of a Lie group $G$. Then there exists a bijection between the invariant Finsler metric $F$ on $G / H$ and the Minkowski norm $F_{0}$ on $\mathfrak{g} / \mathfrak{h}$, such that $\left(\mathfrak{g} / \mathfrak{h}, A d, F_{0}\right)$ is a Minkowski representation of $H$, where $\mathfrak{g}$ and $\mathfrak{h}$ are the Lie algebras of $G$ and $H$ and $\mathrm{Ad}$ is the adjoint action of $H$ on $\mathfrak{g} / \mathfrak{h}$.

Does a given homogeneous manifold admit an invariant non-Riemannian Finsler metric? In [Deng and Hou 2004a], we proved that it does if the adjoint action of $H$ on $\mathfrak{g} / \mathfrak{h}$ is not irreducible. When the adjoint action is irreducible, the situation is more complicated, and in general, a necessary and sufficient condition seems to be unattainable. However, we can give a complete answer when the action of $H$ on $\mathfrak{g} / \mathfrak{h}$ is polar. Let us first recall some definitions in the next section.

\section{Polar actions of Lie groups}

Let $G$ be a compact Lie group with Lie algebra $\mathfrak{g}$ and a real representation $(\rho, V)$. By Weyl's unitary trick, there is an inner product $\langle\cdot, \cdot\rangle$ that is invariant under $\rho(g)$ for all $g \in G$. Therefore, we get a continuous homomorphism $\rho$ from $G$ to $O(V)$, where $O(V)$ is the orthogonal group with respect to $\langle\cdot, \cdot\rangle$. In many situations, we hope to find a linear subspace of $V$ that intersects every orbit of the $G$-action and is of minimal possible dimension. In studying $G$-invariant differential equations or differential operators, such a subspace can be used for reduction of variables. It is also useful in analyzing orbit structure, which is important in geometry.

The existence of such a cross section comes from a simple fact: As pointed out in [Dadok 1985], if for $v \in V$ we let $a_{v}=\{u \in V \mid\langle u, \mathfrak{g} \cdot v\rangle=0\}=(\mathfrak{g} \cdot v)^{\perp}$, then the linear space $a_{v}$ intersects every $G$-orbit. The action of $\mathfrak{g}$ on $V$ is the differential of that of $G$, and hence $\mathfrak{g} \cdot v$ is just the tangent space to the $G$-orbit through $v$. To obtain a cross section of minimal dimension, we only need to choose $v$ on an orbit of maximal dimension. A vector $v$ in $V$ is called regular if $\mathfrak{g} \cdot v$ is of maximal possible dimension. In some special cases, we may choose a cross section that intersects each orbit orthogonally.

Definition 2.1 [Dadok 1985]. A representation $\rho: G \rightarrow O(V)$ is called polar if it satisfies any of the following equivalent conditions:

- For any regular elements $v_{1}$ and $v_{2}$, we have $\mathfrak{g} \cdot v_{1}=k \cdot\left(\mathfrak{g} \cdot v_{2}\right)$ for some $k \in G$.

- For any regular elements $v_{1}$ and $v_{2}$, we have $a_{v_{1}}=k \cdot a_{v_{2}}$ for some $k \in G$.

- For any regular element $v \in V$ and $u \in a_{v}$, the scalar product $\left\langle\mathfrak{g} \cdot u, a_{v}\right\rangle$ vanishes. 
For a polar representation $(\rho, V)$ of $G$, any minimal linear cross section $a_{v}$ is called a Cartan subspace. The dimension of $\mathfrak{a}$ is called the (generalized) rank of the representation.

The notion of a polar representation is closely related to that of a polar action of a compact Lie group on a Riemannian manifold. An isometric action of a compact Lie group $G$ on a Riemannian manifold $M$ is called polar if there exists a closed, connected submanifold $\Sigma$ of $M$ that meets all $G$-orbits and meets these orbits orthogonally. Any such $\Sigma$ is called a section of the action. A section is necessarily totally geodesic in $M$. If the section is flat in the induced Riemannian metric, then the action is called hyperpolar. Therefore, a polar representation is just a hyperpolar action consisting of linear isometries on a Euclidean space.

Now we recall some known results and terminology of polar representations. Let $\mathfrak{a}$ be a fixed Cartan subspace, and let $N_{G}(\mathfrak{a})$ and $Z_{G}(\mathfrak{a})$ be respectively the normalizer and centralizer of $\mathfrak{a}$ in $G$ :

$$
N_{G}(\mathfrak{a})=\{g \in G \mid \rho(g)(\mathfrak{a}) \subset \mathfrak{a}\}, \quad Z_{G}(\mathfrak{a})=\{g \in G \mid \rho(g)(X)=X \text { for } X \in \mathfrak{a}\} .
$$

Then the Lie algebras of $N_{G}(\mathfrak{a})$ and $Z_{G}(\mathfrak{a})$ coincide by [Dadok 1985]. Hence $W=N_{G}(\mathfrak{a}) / Z_{G}(\mathfrak{a})$ is a finite group acting on $\mathfrak{a}$. $W$ is called the Weyl group of the representation.

Theorem 2.2 [Dadok 1985]. Let $\rho: G \rightarrow O(V)$ be a polar representation, and let $\mathfrak{a}$ be a Cartan subspace. Then for any $x \in V$, the orbit $G \cdot x$ intersects $\mathfrak{a}$ at finitely many points and the set of intersections comprises a single $W$-orbit.

Definition 2.3. A symmetric space representation of a connected compact Lie group $G$ (with Lie algebra $\mathfrak{g}$ ) is an orthogonal representation $\rho: G \rightarrow \mathrm{SO}(V)$ such that there exists a noncompact real Lie algebra $\mathfrak{g}_{1}$ with a Cartan decomposition $\mathfrak{g}_{1}=\mathfrak{k}_{1}+\mathfrak{m}_{1}$, a Lie algebra isomorphism $A: \mathfrak{g} \rightarrow \mathfrak{k}_{1}$, and a real vector space isomorphism $L: V \rightarrow \mathfrak{m}_{1}$ such that $L \circ \rho(X)(y)=[A(X), L(y)]$ for all $X \in \mathfrak{g}$ and $y \in V$.

Theorem 2.4 [Dadok 1985, Proposition 6]. Let $\rho: G \rightarrow \mathrm{SO}(V)$ be a polar representation of a connected compact Lie group $G$. Then there exist a connected compact Lie group $G_{1}$ and a symmetric space representation $\rho_{1}: G_{1} \rightarrow \mathrm{SO}(V)$ such that the $G$ - and $G_{1}$-orbits coincide.

Remark 2.5. The fact that the $G$ - and $G_{1}$-orbits coincide does not mean that they are equivalent representations. In fact, the classification of polar representations implies there are irreducible polar representations that are not equivalent to the isotropic representation of a Riemannian symmetric space; see Section 6.

The following result will be useful in proving the main results of Section 3. 
Proposition 2.6. Let $\rho: G \rightarrow \mathrm{SO}(V)$ be a symmetric space representation of a connected compact Lie group $G$. On the vector space $\overline{\mathfrak{g}}=\mathfrak{g}+V$ (direct sum), there is a Lie algebra structure that makes $\overline{\mathfrak{g}}$ into a noncompact semisimple Lie algebra and such that $\overline{\mathfrak{g}}=\mathfrak{g}+V$ is a Cartan decomposition. Further, there exists a Riemannian globally symmetric space $\bar{G} / \bar{K}$ of noncompact type such that $\overline{\mathfrak{g}}=\mathfrak{g}+V$ is the canonical decomposition of the corresponding Lie algebra, and the G-orbit in $V$ coincides with the $\bar{K}$-orbit in $V$ of the adjoint representation of $\bar{K}$ on $V$.

Proof. Let $\mathfrak{g}_{1}, \mathfrak{k}_{1}, \mathfrak{m}_{1}, A, L$ be as in Definition 2.3. In the vector space $\overline{\mathfrak{g}}=\mathfrak{g}+V$ (where the addition is direct sum of subspaces), we introduce a bracket: On $\mathfrak{g}$ we take the same bracket operation as that of the Lie algebra. For $x, y \in V$, the bracket is equal to $A^{-1}([L(x), L(y)])$, where the Lie bracket is the same as that of $\mathfrak{g}_{1}$. (Note that $L(x), L(y)$ are contained in $\mathfrak{m}_{1}$, so $[L(x), L(y)]$ lies in $\mathfrak{k}_{1}$ and $A^{-1}([L(x), L(y)])$ is contained in $\mathfrak{g}$.) For $X \in \mathfrak{g}, x \in V$, we define $[X, x]=L^{-1}([A(X), L(x)])$, which is an element in $V$. It can be checked directly (albeit nontrivially) that $\overline{\mathfrak{g}}$ with the brackets above forms a Lie algebra that is isomorphic to $\mathfrak{g}_{1}$. Since $\mathfrak{g}_{1}$ is a noncompact semisimple Lie algebra, so is $\overline{\mathfrak{g}}$, and since $\mathfrak{g}_{1}=\mathfrak{k}_{1}+\mathfrak{m}_{1}$ is a Cartan decomposition, so is $\overline{\mathfrak{g}}=\mathfrak{g}+V$. Now, according to the theory of orthogonal symmetric Lie algebras [Helgason 1978], there exists a Riemannian symmetric pair $(\bar{G}, \bar{K})$ of noncompact type with Lie $\bar{G}=\overline{\mathfrak{g}}$ and Lie $\bar{K}=\mathfrak{g}$. Moreover, by the definition of the brackets, the differential of the adjoint representation of $\bar{K}$ on $V$ is just the induced action of $\mathfrak{g}$ on $V$ of the representation $\rho$ of $G$. Since $\rho$ is an orthogonal representation, the inner product $\langle\cdot, \cdot\rangle$ on $V$ is invariant under the action of $G$. Hence, $\rho(X)$ is skew-symmetric with respect to $\langle\cdot, \cdot\rangle$ for any $X \in \mathfrak{g}$. Since in the Riemannian symmetric pair of noncompact type, the subgroup must be compact and connected [Helgason 1978], $\bar{K}$ must be a connected compact Lie group. This implies that the exponential map of $\mathfrak{g}$ to $\bar{K}$ must be surjective [Kobayashi and Nomizu 1963]. Thus $\langle\cdot, \cdot\rangle$ is also invariant under the action of $\bar{K}$. Hence, there is a $G_{2}$-invariant Riemannian metric $Q$ on $G_{2} / K_{2}$ whose restriction on $T_{o}(\bar{G} / \bar{K})=V$ is $\langle\cdot, \cdot\rangle$, where $o$ is the origin of $\bar{G} / \bar{K}$. Then $(\bar{G} / \bar{K}, Q)$ is a Riemannian globally symmetric space [Helgason 1978]. Now the proposition follows from the facts that the exponential map of $G$ is surjective, and that the differentials of $\rho$ and of the adjoint representation of $K_{2}$ on $V$ coincide.

\section{Classification of Minkowski representations associated with a polar representation}

In the two theorems below, let $G$ be a compact connected Lie group with a polar representation $(V, \rho)$.

Theorem 3.1. Let $\mathfrak{a} \subset V$ be a Cartan subspace and $W$ be the corresponding Weyl group. Then there exists a bijection between the set of Minkowski norms on $V$ that 
make $(V, \rho, F)$ a Minkowski representation, and the set of $W$-invariant Minkowski norms on $\mathfrak{a}$.

Theorem 3.2. - If the generalized rank of $(V, \rho)$ is 1 , then there does not exist a non-Euclidean Minkowski norm $F$ on $V$ such that $(V, \rho, F)$ is a Minkowski representation of $G$.

- If the generalized rank of $(V, \rho)$ is at least 2 , then there exist infinitely many non-Euclidean Minkowski norms $F$ on $V$ such that $(V, \rho, F)$ is a Minkowski representation of $G$.

We remark here that Szabó's argument [1981] on the existence of invariant nonRiemannian Finsler metrics on a Riemannian symmetric space is also valid for polar homogeneous space, since on a polar homogeneous space the isotropic representation has the same orbit as that of a Riemannian symmetric space. Hence Theorem 3.2 should not be viewed as a new result. The main point here is that our refinement of Szabó's argument can be used to establish the bijection stated in Theorem 3.1. This will lead to a classification of all the invariant Finsler metrics on a polar homogeneous space; see Section 4.

To prove the two theorems above, we need several lemmas.

Lemma 3.3. Let $G,(V, \rho), \mathfrak{a}$ and $W$ be as in Theorem 3.1. Then any $W$-invariant Minkowski norm on $\mathfrak{a}$ can be uniquely extended to a $G$-invariant functional on $V$ that is smooth on $V \backslash\{0\}$.

Proof. We use the same argument as in [Szabó 2006]. Since $\mathfrak{a}$ is a Cartan subspace, according to Definition 2.1, a intersects every orbit of the action of $G$ on $V$. Thus for any $y \in V$, there exist a $y_{a} \in \mathfrak{a}$ and $g_{y} \in G$ (not necessarily unique) such that $g_{y}\left(y_{a}\right)=y$. We now define a functional $L$ on $V$ by $L(y)=F\left(y_{a}\right)$. Since $F$ is $W$-invariant, it is easy to check that $L$ is well defined. To prove that $L$ is smooth on the slit space $V \backslash\{0\}$, we need a result of [Dadok 1982], which says that the extension of a smooth $W$-invariant function on $\mathfrak{a}$ to $V$ is also smooth. The Minkowski norm $F$ is only smooth on $\mathfrak{a} \backslash\{0\}$ but is continuous on the whole space $\mathfrak{a}$. Define a functional $F_{1}$ by

$$
F_{1}(y)=e^{-1 /\langle y, y\rangle} \cdot F(y) \quad \text { for } y \in V \backslash\{0\} ; \quad F_{1}(0)=0,
$$

where $\langle\cdot, \cdot\rangle$ is the inner product on $\mathfrak{a}$. Then $F_{1}$ is smooth on all of $\mathfrak{a}$. Since $\rho$ is an orthogonal representation, $\langle\cdot, \cdot\rangle$ is $G$-invariant. Thus $F_{1}$ is also $W$-invariant. Also, the extension of $F_{1}$ to $V$ is equal to $e^{-1 /\langle X, X\rangle} F(X)$ on $\mathfrak{g} \backslash\{0\}$. From this the smoothness of $F$ on $\mathfrak{g} \backslash\{0\}$ follows. The uniqueness of the extension is obvious.

Lemma 3.3 establishes the smoothness of the extension of the $W$-invariant Minkowski norms. Next we consider the strong convexity of the extension. Since it is very difficult to obtain strong convexity directly, we first prove a lemma about strict 
convexity. For this we need some results related to Kostant's celebrated convexity theorem, whose theory we now sketch.

Let $(G / H, Q)$ be a globally Riemannian manifold, and let $(\mathfrak{g}, \sigma)$ be the corresponding orthogonal Lie algebra. Let $\mathfrak{g}=\mathfrak{h}+\mathfrak{p}$ be the canonical decomposition of the orthogonal Lie algebra. Then we can identify the tangent space $T_{e H}(G / H)$ of $G / H$ at the origin with the space $\mathfrak{p}$. The isotropic representation of $H$ on $T_{e H}(G / H)$ then corresponds to the adjoint representation of $H$ on $\mathfrak{p}$. Let $\mathfrak{t}$ be a maximal commutative subspace in $\mathfrak{p}$. Then $t$ is a cross section of the action of $H$ and it intersects every orbit orthogonally. Therefore the isotropic representation is polar. Let $W$ be the corresponding Weyl group and $\pi$ be the orthogonal projection of $\mathfrak{p}$ onto $\mathfrak{t}$.

Kostant's convexity theorem [1973]. For any point $x \in \mathfrak{t}$, the subset $\pi(H \cdot x)$ is equal to the convex hull of the points $W \cdot x$, where $H \cdot x$ is the orbit of the point $x$.

To prove the strong convexity of the extension, we still need a lemma on the convexity of the orbit of a convex domain in $V$. Let $\mathfrak{g}$ be a noncompact semisimple Lie algebra, let $\mathfrak{g}=\mathfrak{k}+\mathfrak{p}$ be a Cartan decomposition of $\mathfrak{g}$, and let $\mathfrak{t}$ be a maximal commutative subspace of $\mathfrak{p}$. Let $W_{\mathfrak{t}}$ be the corresponding Weyl group and $\bar{C}$ be a fixed Weyl chamber in $\mathfrak{t}$. The Cartan-Killing form $B$ of $\mathfrak{g}$ is positive definite on $\mathfrak{p}$, so $\langle x, y\rangle=B(x, y)$ defines an inner product on $\mathfrak{p}$. The restriction of this inner product to $\mathfrak{t}$, which we still denote by $\langle\cdot, \cdot\rangle$, is $W_{\mathrm{t}}$-invariant. The dual cone of $\bar{C}$, denoted by $\bar{C}^{*}$, is defined by $x \in \bar{C}^{*}$ if and only if $\langle x, y\rangle \geq 0$ for all $y \in \bar{C}$. A partial order can be defined on $\mathfrak{t}$ such that $x \geq y$ if and only if $x-y \in \bar{C}^{*}$. Then Kostant [1973] proved that $x \leq y$ for $x, y \in \bar{C}$ if and only if $x$ lies in the convex hull of the $W$-orbit of $y$. Let $H$ be the maximal compact subgroup of the adjoint group Int $\mathfrak{g}$ of $\mathfrak{g}$. It is well known that each $H$-orbit in $\mathfrak{g}$ intersects $\bar{C}$ at exactly one point [Helgason 1978]. For $x \in \mathfrak{p}$, we denote by $\bar{C}(x)$ the unique element of the intersection of the orbit $G \cdot x=\{g \cdot x \mid g \in G\}$ and $\bar{C}$.

The following result is proved using Kostant's convexity theorem.

Theorem 3.4 [Tam 1998]. For any $x, y \in \mathfrak{p}$, we have $\bar{C}(x+y) \leq \bar{C}(x)+\bar{C}(y)$.

The following lemma will be useful in proving the main result of this paper.

Lemma 3.5. Let $D$ be a strictly convex domain in $\mathfrak{t}$ containing the origin, with smooth boundary $S$, and let $D$ be invariant under the action of $W_{\mathrm{t}}$. Then the orbit of $D$ under the action of $H$ forms a strictly convex domain in $\mathfrak{p}$.

Proof. Since $D$ is $W$-invariant, the boundary $S$ is also $W_{\mathrm{t}}$-invariant. Define a nonnegative function $h_{1}$ on $\mathfrak{t}$ by $h_{1}(y)=1 / t$ where $t>0$ is such that $t y \in S$. Then $h_{1}$ is smooth on $\mathfrak{t} \backslash\{0\}$, and $h_{1}(\lambda y)=\lambda h_{1}(y)$ for any $\lambda>0$. Also, $h_{1}$ satisfies the triangle inequality: $h_{1}(x+y) \leq h_{1}(x)+h_{1}(y)$ with the equality holding if and only if $x=\alpha y$ or $y=\alpha x$ for some $\alpha \geq 0$ [Bao et al. 2000]. Moreover, the function $h_{1}$ 
is obviously $W_{\mathrm{t}}$-invariant. Hence $h_{1}$ can be extended to a well-defined function $h_{2}$ on $\mathfrak{p}$ by defining $h_{2}(g \cdot y)=y$ for $g \in H$ and $y \in \mathfrak{t}$. Then it is easily seen that $h_{2}$ is $H$-invariant and the orbit of $D$ forms the set $D_{1}=\left\{y \in \mathfrak{g} \mid h_{2}(y)<1\right\}$. Now suppose $y_{1}, y_{2} \in \bar{D}_{1}$ and $0 \leq \lambda \leq 1$. Let $g \in H$ be such that

$$
g \cdot\left(\lambda y_{1}+(1-\lambda) y_{2}\right)=\bar{C}\left(\lambda y_{1}+(1-\lambda) y_{2}\right) .
$$

Then we have

$$
h_{2}\left(\lambda y_{1}+(1-\lambda) y_{2}\right)=h_{2}\left(g \cdot\left(\lambda y_{1}+(1-\lambda) y_{2}\right)\right)=h_{1}\left(\bar{C}\left(\lambda y_{1}+(1-\lambda) y_{2}\right)\right) .
$$

Suppose $W_{\mathfrak{t}}=\left\{w_{1}, w_{2}, \ldots, w_{s}\right\}$, where $s=\left|W_{\mathfrak{t}}\right|$. Then by Theorem 3.4 and Kostant's convexity theorem, there exist nonnegative numbers $\alpha_{i}$ for $i=1,2, \ldots, s$ that sum to one and satisfy

Hence

$$
\bar{C}\left(\lambda y_{1}+(1-\lambda) y_{2}\right)=\sum_{i=1}^{s} \alpha_{i} w_{i}\left(\bar{C}\left(\lambda y_{1}\right)+\bar{C}\left((1-\lambda) y_{2}\right)\right) .
$$

$$
\begin{aligned}
& h_{1}\left(\bar{C}\left(\lambda y_{1}+(1-\lambda) y_{2}\right)\right) \\
& =h_{1}\left(\sum_{i=1}^{s} \alpha_{i} w_{i}\left(\bar{C}\left(\lambda y_{1}\right)+\bar{C}\left((1-\lambda) y_{2}\right)\right)\right) \leq \sum_{i=1}^{s} h_{1}\left(\alpha_{i} w_{i}\left(\bar{C}\left(\lambda y_{1}\right)+\bar{C}\left((1-\lambda) y_{2}\right)\right)\right) \\
& =\sum_{i=1}^{s} \alpha_{i} h_{1}\left(w_{i}\left(\bar{C}\left(\lambda y_{1}\right)+\bar{C}\left((1-\lambda) y_{2}\right)\right)\right)=\sum_{i=1}^{s} \alpha_{i} h_{1}\left(\left(\bar{C}\left(\lambda y_{1}\right)+\bar{C}\left((1-\lambda) y_{2}\right)\right)\right) \\
& \leq \sum_{i=1}^{s} \alpha_{i}\left(h_{1}\left(\bar{C}\left(\left(\lambda y_{1}\right)\right)+h_{1}\left(\bar{C}(1-\lambda) y_{2}\right)\right)\right)=\sum_{i=1}^{s} \alpha_{i}\left(h_{2}\left(\lambda y_{1}\right)+h_{2}\left((1-\lambda) y_{2}\right)\right) \\
& =\sum_{i=1}^{s} \alpha_{i}\left(\lambda h_{2}\left(y_{1}\right)+(1-\lambda) h_{2}\left(y_{2}\right)\right)=\lambda h_{2}\left(y_{1}\right)+(1-\lambda) h_{2}\left(y_{2}\right) \leq \lambda+(1-\lambda)=1 .
\end{aligned}
$$

Thus $\lambda y_{1}+(1-\lambda) y_{2} \in \bar{D}_{1}$. Further, if $h_{2}\left(\lambda y_{1}+(1-\lambda) y_{2}\right)=1$, then from the above equation we see that either $h_{2}\left(y_{1}\right)=1$ with $\lambda=1$ or $h_{2}\left(y_{2}\right)=1$ with $\lambda=0$; that is, either $\lambda y_{1}+(1-\lambda) y_{2}=y_{1}$ or $\lambda y_{1}+(1-\lambda) y_{2}=y_{2}$. Hence the interior of the line segment joining $y_{1}$ and $y_{2}$ is contained in $D_{1}$. This proves the lemma.

Corollary 3.6. Let $G, V, \rho$ and $\mathfrak{a}$ be as in Theorem 3.1. Let $D$ be a strictly convex domain in $\mathfrak{a}$ containing the origin and invariant under the action of the Weyl group. Then the orbit of $D$ under the action of $G$ forms a strictly convex domain in $V$.

Proof. By Dadok's result, the $G$-orbit coincides with that of a symmetric space representation $\rho_{1}: G \rightarrow \mathrm{SO}(V)$. Then by Proposition 2.6, we can assume that $\mathfrak{a}$ is a Cartan subspace of a Riemannian symmetric space of the noncompact type, and that the action $\rho_{1}$ of $G$ on $V$ is exactly the isotropic representation of the Riemannian symmetric space. Now the corollary follows from Lemma 3.5. 
Proof of Theorem 3.1. If $F$ is a Minkowski norm on $V$ such that $(V, \rho)$ is a Minkowski representation of $G$, then $\left.F\right|_{\mathfrak{a}}$ is a $W$-invariant function and obviously a Minkowski norm on $\mathfrak{a}$. It is a direct consequence of the definition of the Cartan subspace that this correspondence is one-to-one. To prove it is surjective, let $F_{1}$ be a $W$-invariant Minkowski norm on $\mathfrak{a}$. For any $x \in V$, there exists $g \in G$ such that $\rho(g)(x) \in V$. We then define a function $F$ on $V$ by $F(x)=F_{1}(\rho(g)(x))$. Since $F_{1}$ is $W$-invariant, $F$ is well defined. By Lemma 3.3, $F$ is smooth on $V \backslash\{0\}$. Next we prove that $F$ is a Minkowski norm on $V$. Let $\alpha_{1}, \ldots, \alpha_{m}$ be an orthonormal basis of $\mathfrak{a}$ with respect to the inner product restricted to $\mathfrak{a}$, and write $F_{1}(z)=$ $F_{1}\left(z^{1}, z^{2}, \ldots, z^{n}\right)$ for $z=\sum_{i=1}^{m} z^{i} \alpha_{i}$. Then we define the Hessian matrix of $F_{1}$ by [Bao et al. 2000]

$$
\left(a_{i j}\right)=\left(\left[\frac{1}{2} F_{1}^{2}\right]_{z^{i} z^{j}}\right) .
$$

For any $y \neq 0$, denote the minimal eigenvalue of the matrix $\left(a_{i j}(y)\right)$ by $\mu(y)$. Let

$$
\mu=\inf _{\{y \in \mathfrak{a} \mid\langle y, y\rangle=1\}} \mu(y) .
$$

Since $F_{1}$ is a Minkowski norm, $\mu(y)>0$ for any $y \in \mathfrak{a} \backslash\{0\}$. Since $\{y \in \mathfrak{a} \mid\langle y, y\rangle=1\}$ is compact and the function $\mu(y)$ is continuous, we have $\mu>0$. Now on $\mathfrak{a}$ we write

$$
F_{1}(x)=\sqrt{\left(F_{1}^{2}(x)-\frac{1}{2} \mu\langle x, x\rangle\right)+\frac{1}{2} \mu\langle x, x\rangle}=\sqrt{L_{*}(x)+\frac{1}{2} \mu\langle x, x\rangle},
$$

where $L_{*}(x)=F_{1}^{2}(x)-\frac{1}{2} \mu\langle x, x\rangle$. Since $F_{1}^{2}(x)=\sum_{i, j=1}^{n} a_{i j}(x) x^{i} x^{j}$ for $x \in V \backslash\{0\}$, we have

$$
F_{1}^{2}(x) \geq \sum_{i=1}^{n} \mu(x) x^{i} x^{i}=\mu(x)\langle x, x\rangle .
$$

Hence $L_{*}(x)>0$ for any $x \neq 0$. Further, the Hessian matrix of $\sqrt{L_{*}(x)}$ is positive definite at any $x \in \mathfrak{a} \backslash\{0\}$. Hence $\sqrt{L_{*}}$ is also a Minkowski norm on $\mathfrak{a}$. In particular, the domain $\left\{y \in \mathfrak{a} \mid L_{*}(y)<1\right\}$ is strictly convex [Bao et al. 2000]. Denote its boundary by $S$. Since $\langle x, x\rangle$ is $W$-invariant, $L_{*}(x)$ is also $W$-invariant. Therefore $L_{*}(x)$ can be uniquely extended to a functional $L$ on $V$ that is smooth on $V \backslash\{0\}$. By Lemma 3.5, the orbit of $S$ under the action of $G$ is the boundary of a strictly convex domain $D_{1}$ in $V$. But it is obvious that $D_{1}=\{y \in V \mid L(y)<1\}$. Therefore, the Hessian matrix of $\sqrt{L}$ (with respect to certain basis of $V$ ) is positive semidefinite [Bao et al. 2000]. Therefore the Hessian matrix of $F$ is positive definite at any $x \neq 0$. Thus $F$ is a Minkowski norm. This proves that the correspondence above is surjective. Therefore it is a bijection.

Proof of Theorem 3.2. By Theorem 3.1, we only need to consider the $W$-invariant Minkowski norms on a Cartan subspace $\mathfrak{a}$.

If $\operatorname{dim} V=1$, the conclusion is obvious. Therefore, we suppose that $\operatorname{dim} V \geq 2$. 
If the generalized rank of the polar representation is 1 , then we assert that the Weyl group $W$ consists of two elements: $W=\{1,-1\}$. In fact, since the Weyl group is generated by reflections, we have only two possibilities: either $W=\{1\}$ or $W=\{1,-1\}$. If $W=\{1\}$, each $W$-orbit consists of only one point. Consider the unit sphere $S$ of $V$, and let $u$ and $-u$ be the unit element in $\mathfrak{a}$. Since each $G$-orbit intersects $\mathfrak{a}$ at one point, we have $S=G \cdot u \cup G \cdot(-u)$ and $G \cdot u \cap G \cdot(-u)=\varnothing$. Now a contradiction arises, because by the theory of Lie transformation groups, the orbits $G \cdot u$ and $G \cdot(-u)$ are connected closed submanifolds of $S$ [Helgason 1978]. Hence $W=\{1,-1\}$. Suppose $F$ is a $W$-invariant Minkowski norm on $\mathfrak{a}$. Then we have $F(x)=F(-x)$. Since $\operatorname{dim} \mathfrak{a}=1$, we see that $F$ is a Euclidean norm on $\mathfrak{a}$. Suppose $F(x)=c \sqrt{\langle x, x\rangle}$ for $x \in \mathfrak{a}$, where $\langle\cdot, \cdot\rangle$ is the $G$-invariant inner product on $V$ and $c$ is a positive constant. Then by Theorem 3.1, the extension of $F$ to $V$ must be equal to $c \sqrt{\langle\cdot, \cdot\rangle}$ on $V$. This proves the first conclusion of the theorem.

Now we suppose that the generalized rank of the polar action is at least 2. By Dadok's result and Proposition 2.6, there exists a Riemannian symmetric space $G_{1} / H_{1}$ of noncompact type with canonical decomposition $\mathfrak{g}_{1}=\mathfrak{h}_{1}+\mathfrak{p}_{1}$ such that $V$ is linearly isometric to $\mathfrak{p}_{1}$ through a linear isometry $\tau$, and such that the $G$ orbit corresponds to the $H_{1}$-orbit of the isotropic representation on $\mathfrak{p}_{1}$ through $\tau$. It is easily seen that $\mathfrak{a}_{1}=\tau(\mathfrak{a})$ is a maximal abelian subalgebra (that is, a Cartan subspace of the polar action of $H_{1}$ on $\mathfrak{p}_{1}$ ) of $\mathfrak{p}_{1}$. Let $W_{1}$ be the corresponding Weyl group. Then $W$-orbits correspond to $W_{1}$-orbits. Hence, to prove the theorem in this case, we only need to prove that there are infinitely many $W_{1}$-invariant Minkowski norms on $\mathfrak{a}_{1}$. Fix one Weyl chamber, say $\mathscr{C}$. It is known that the closure $\overline{\mathscr{C}}$ of $\mathscr{C}$ is a fundamental domain of the action of $W_{1}$. That is, every orbit intersects $\overline{\mathscr{C}}$ at exactly one point. Now we assert that there exist infinitely many functions $f$ on $\mathscr{C}$ such that

- $f(\lambda x)=\lambda f(x)$ for all $\lambda>0$,

- $f$ can be extended to a $W$-invariant smooth function $f_{1}$ on $\mathfrak{a}_{1} \backslash\{0\}$, and

- the domain $\left\{x \in \mathfrak{a}_{1} \mid f_{1}(x)<1\right\}$ is strictly convex.

For example, we first choose a sphere (centered at the origin) with respect to the inner product on $\mathfrak{a}_{1}$. Then we choose a hyperplane whose the intersection with the sphere is contained in $\mathscr{C}$. In this way, we get a hypersurface $S_{1}$, which, together with the Weyl walls of $\mathscr{C}$, bounds a strictly convex domain. The hypersurface $S_{1}$ is of course not smooth, but it is easily seen that we can make it smooth, while keeping the bounded domain strictly convex. We denote one such hypersurface by $S$ and define a function $f(x)$ on $\mathscr{C}$ by $f(x)=\lambda$ if $x / \lambda \in S$. Then $f(x)$ satisfies the conditions above. It is obvious that there exist infinitely many functions of this type. Now, each such $f$ can be extended to a function $F_{1}$ on $\mathfrak{a}_{1}$ and, similarly to 
the proof of Theorem 3.1, we see that the function

$$
F(u)=\sqrt{F_{1}^{2}(u)+\langle u, u\rangle},
$$

with $u \in \mathfrak{a}_{1}$, defines a $W_{1}$-invariant Minkowski norm on $\mathfrak{a}_{1}$.

\section{Invariant Finsler metrics: A classification}

Let $G$ be a Lie group and $H$ be a closed subgroup of $G$. Then on the coset space $G / H$ there exists a smooth structure such that $G$ becomes a Lie transformation group of $G / H$. A fundamental problem in geometry to study the $G$-invariant geometric structures on $G / H$. In [Deng and Hou 2004a], we considered this problem for Finsler metrics. We proved that there is a one-to-one correspondence between the $G$-invariant Finsler metrics on $G / H$ and the $H$-invariant Minkowski norms on the tangent space $T_{o}(G / H)$ of $G / H$ at the origin $o=e H$. Therefore, to classify the $G$-invariant Finsler metrics, we only need to classify the Minkowski representations of $H$ on $T_{o}(G / H)$.

Without losing generality, we can assume that $H$ is a compact subgroup of $G$. In fact, if $(M, F)$ is a connected homogeneous Finsler space, then the isotropic subgroup (at a fixed point $x \in M)$ of the full group $I_{x}(M, F)$ of isometries $I(M, F)$ must be a compact subgroup of $I(M, F)$ by [Deng and Hou 2002]. Hence $M=$ $I(M, F) / I_{0}(M, F)$ and $F$ can be viewed as an $I(M, F)$-invariant Finsler metric on $M$. The compactness of $H$ implies that there exist $H$-invariant inner products on the tangent space $T_{o}(G / H)$. Fix one such inner product, and denote it by $\langle\cdot, \cdot\rangle$. Then $\langle\cdot, \cdot\rangle$ can be extended to $\mathfrak{g}=\operatorname{Lie} G$ so that

$$
\langle\operatorname{Ad}(h)(x), \operatorname{Ad}(h)(y)\rangle=\langle x, y\rangle \quad \text { for all } h \in H \text { and } x, y \in \mathfrak{g} .
$$

Let $\mathfrak{m}$ be the orthogonal complement of $\mathfrak{h}=$ Lie $H$. Then $\mathfrak{m}$ satisfies

$$
\operatorname{Ad}(h) \mathfrak{m} \subset \mathfrak{m} \quad \text { and } \quad \mathfrak{g}=\mathfrak{h}+\mathfrak{m} .
$$

Hence the tangent space $T_{o}(G / H)$ can be identified with $\mathfrak{m}$, and the isotropic representation of $H$ on $T_{o}(G / H)$ corresponds to the adjoint representation of $H$ on $\mathfrak{m}$.

Our goal is to a classify all the $G$-invariant Finsler metrics on $G / H$. We stress here that this problem for Riemannian metrics is easy. In fact, since $H$ is compact, $\mathfrak{m}$ can be decomposed into a direct sum of subspaces

$$
\mathfrak{m}=\mathfrak{m}_{0}+\mathfrak{m}_{1}+\cdots+\mathfrak{m}_{k},
$$

where $\mathfrak{m}_{0}$ consists of $H$-fixed vectors in $\mathfrak{m}$, and $\mathfrak{m}_{i}$ for $i=1,2, \ldots, k$ are invariant, irreducible subspaces of $H$. For simplicity, we assume that the submodules $m_{i}$ are not equivalent to each other. If there are two $H$-invariant inner products on $\mathfrak{m}$, 
then by Schur's lemma, for each $i$ with $1 \leq i \leq k$, the restrictions of the two inner products to $\mathfrak{m}_{i}$ must differ only by a positive multiple. On the other hand, any inner product is $H$-invariant on $\mathfrak{m}_{0}$. Fix an $H$-invariant inner product $\langle\cdot, \cdot\rangle_{i}$ on $\mathfrak{m}_{i}$ for $i=1,2, \ldots, k$. Then any $H$-invariant inner product must be of the form

$$
\langle\cdot, \cdot\rangle_{0}+c_{1}\langle\cdot, \cdot\rangle_{1}+\cdots+c_{k}\langle\cdot, \cdot\rangle_{k},
$$

where $\langle\cdot, \cdot\rangle_{0}$ is an arbitrary inner product on $\mathfrak{m}_{0}$, and $c_{1}, c_{2}, \ldots, c_{k}$ are arbitrary positive real numbers. This classifies $H$-invariant inner products on $\mathfrak{m}$, and hence $G$-invariant Riemannian metrics on $G / H$.

Therefore, the difficult case is that of non-Riemannian Finsler metrics. Since the general problem seems to be unsolvable, we restrict to the special case where the isotropic representation is polar. Theorems 3.1 and 3.2 give this:

Theorem 4.1. Let $G$ be a Lie group and $H$ be a compact subgroup of $G$. Suppose that the isotropic representation of $G / H$ at the origin $o=e H$ is polar and $\mathfrak{m}$ is as in Equation (4-1).

- If the generalized rank of the adjoint action of $H$ on $\mathfrak{m}$ is 1 , then there does not exist any non-Riemannian invariant Finsler metric on $G / H$.

- If the generalized rank of the action of $H$ on $\mathfrak{m}$ is at least 2 , then there exist infinitely many non-Riemannian invariant Finsler metrics on $G / H$ (even if we do not distinguish those metrics that are differ only by a positive multiple). In this case, there is a one-to-one correspondence between the G-invariant Finsler metrics on $G / H$ and the $W$-invariant Minkowski norms on $\mathfrak{a}$, where $\mathfrak{a}$ is a Cartan subspace of $\mathfrak{m}$ and $W$ is the corresponding Weyl group.

Now we consider some special cases of Theorem 4.1. Suppose $(G, H)$ is a Riemannian symmetric pair. That is, $G$ is a connected Lie group and $H$ is a closed subgroup of $G$ such that there exists an involutive automorphism $\sigma$ such that $\left(G_{\sigma}\right)_{e} \subset H \subset G_{\sigma}$, where $G_{\sigma}$ denotes the fixed points of $\sigma$ and $\left(G_{\sigma}\right)_{e}$ denotes its unit components. Suppose also that the isotropic action of $H$ at $T_{o}(G / H)$ leaves certain inner products invariant. We consider the classification of all $G$-invariant Finsler metrics on $G / H$. Note that in this case the isotropic action is polar.

Theorem 4.2. Let $(G, H)$ be a Riemannian symmetric pair. Let $\mathfrak{g}=\mathfrak{h}+\mathfrak{m}$ be the canonical decomposition of the Lie algebra of $G$. Fix one maximal subspace $\mathfrak{a}$ of $\mathfrak{m}$, and let $W$ be the corresponding Weyl group. Then there exists a bijection between the $G$-invariant Finsler metrics on $G / H$ and the $W$-invariant Minkowski norms on $\mathfrak{a}$. In particular, if the rank of $G / H$ is 1 , there does not exist any $G$ invariant non-Riemannian Finsler metric on $G / H$; if the rank of $G / H$ is at least 2, there exist infinitely many $G$-invariant non-Riemannian Finsler metrics on $G / H$. 
The existence part of this theorem was established in [Szabó 1981], but the one-to-one part was not given there.

By [Deng and Hou 2007], any $G$-invariant Finsler metric on $G / H$ is a globally symmetric Berwald metric; and by [Deng and Hou 2005a], any globally symmetric Berwald metric can be constructed in this way. Therefore, Theorem 4.2 can be viewed as a classification of all globally symmetric Berwald metrics.

Example 4.3. Consider the unit sphere $S^{n}$ in Euclidean $\mathbb{R}^{n+1}$. It is a Riemannian symmetric space of rank 1 . The special orthogonal group $\mathrm{SO}(n+1)$ acts transitively on $S^{n}$, and the isotropic subgroup at $(1,0, \ldots, 0)$ can be identified with the subgroup $\mathrm{SO}(n)$ of $\mathrm{SO}(n+1)$. Thus $S^{n}$ can be viewed as the coset space $\mathrm{SO}(n+1) / \mathrm{SO}(n)$, and the induced Riemannian metric on $S^{n}$ can be viewed as an $\mathrm{SO}(n+1)$-invariant metric on $\mathrm{SO}(n+1) / \mathrm{SO}(n)$. By Theorem 4.2, there is no $\mathrm{SO}(n+1)$-invariant non-Riemannian Finsler metric on $S^{n}$. Now we consider the product manifold $S^{n} \times S^{m}$, where $m, n \geq 1$. Write $S^{n} \times S^{m}$ as

$$
G / H=(\mathrm{SO}(n+1) \times \mathrm{SO}(m+1)) /(\mathrm{SO}(n) \times \mathrm{SO}(m)) .
$$

The rank of $G / H$ is 2 . Therefore there exist infinitely many $G$-invariant nonRiemannian Finsler metrics on $G / H$. All these metrics are globally symmetric and of Berwald type. Now we can give an explicit description of these metrics. Let $\mathfrak{a}_{1}$ and $\mathfrak{a}_{2}$ be Cartan subspaces of $\mathrm{SO}(n+1) / \mathrm{SO}(n)$ and $\mathrm{SO}(m+1) / \mathrm{SO}(m)$, respectively. The corresponding Weyl groups on $\mathfrak{a}_{1}$ and $\mathfrak{a}_{2}$ are $W_{1}=W_{2}=\{1,-1\}$. The direct sum $\mathfrak{a}=\mathfrak{a}_{1} \oplus \mathfrak{a}_{2}$ is a Cartan subspace of $G / H$, and the corresponding Weyl group $W$ consists of four elements: $W=\left\{1, \sigma_{1}, \sigma_{2}, \sigma_{3}\right\}$, where

$\sigma_{1}(x+y)=-x+y, \quad \sigma_{2}(x+y)=x-y, \quad \sigma_{3}(x+y)=-x-y \quad$ for $x \in \mathfrak{a}_{1}, y \in \mathfrak{a}_{2}$.

Therefore a Minkowski norm $F$ on $\mathfrak{a}$ is $W$-invariant if and only if $F$ satisfies

$$
F( \pm x \pm y)=F(x+y) \quad \text { for } x \in \mathfrak{a}_{1}, y \in \mathfrak{a}_{2} .
$$

By Theorem 4.2, there is a one-to-one correspondence between the $G$-invariant Finsler metrics on $S^{n} \times S^{m}$ and the Minkowski norms on $\mathfrak{a}$ satisfying (4-2). It is easily seen that there actually exist infinitely many Minkowski norms on $\mathfrak{a}$ that satisfy (4-2). For example, identifying $\mathfrak{a}_{1}$ and $\mathfrak{a}_{2}$ with $\mathbb{R}^{1}$, we can define a set of Minkowski norms by

$$
F_{\mu}(x+y)=\sqrt{x^{2}+y^{2}+\mu \sqrt{x^{4}+y^{4}}} \text { for } x, y \in \mathbb{R}^{1},
$$

where $\mu$ is an arbitrary positive real number. These norms satisfy (4-2) and are pairwise not mutually linearly isometric [Bao et al. 2000].

Similarly, we can consider other Riemannian symmetric spaces of rank 1 and their product. Next we give an irreducible example of rank at least 2. 
Example 4.4. Consider the Riemannian symmetric pair $(\operatorname{SL}(n, \mathbb{R}), \operatorname{SO}(n))$, where $n \geq 3$. The rank is $n-1$. So there exist infinitely many $\operatorname{SL}(n, \mathbb{R})$-invariant nonRiemannian Finsler metrics on $\operatorname{SL}(n, \mathbb{R}) / \operatorname{SO}(n)$. Now we explicitly describe these metrics. The canonical decomposition of the Lie algebra is $\mathfrak{s l}(n, \mathbb{R})=\mathfrak{s o}(n)+\mathfrak{p}$, where $\mathfrak{p}$ consists of all $n \times n$ traceless symmetric matrices. A Cartan space can be taken as the space of all diagonal matrices in $\mathfrak{p}$, denoted by $\mathfrak{a}$. The corresponding Weyl group is isomorphic to the full permutation group of $n$ indices, which acts on $\mathfrak{a}$ by permuting the entries along the diagonal [Helgason 1978]. Therefore, if we write the elements in $\mathfrak{a}$ as

$$
\operatorname{diag}\left(\lambda_{1}, \lambda_{2}, \ldots, \lambda_{n}\right), \quad \text { where } \sum_{i=1}^{n} \lambda_{i}=0,
$$

then a Minkowski norm $F$ on $\mathfrak{a}$ is $W$-invariant if and only if $F\left(\lambda_{1}, \ldots, \lambda_{n}\right)$ is a symmetric function of $\lambda_{1}, \lambda_{2}, \ldots, \lambda_{n}$. An explicit series of such norms can be constructed as follows:

$$
F_{\mu}\left(\lambda_{1}, \ldots, \lambda_{n}\right)=\sqrt{\sum_{i=1}^{n} \lambda_{i}^{2}+\mu \sqrt{\sum_{i=1}^{n} \lambda_{i}^{4}}},
$$

where $\mu$ is an arbitrary positive real number. As in Example 4.3, the Minkowski norms above define infinitely many $\operatorname{SL}(n, \mathbb{R})$-invariant non-Riemannian Finsler metrics on $\operatorname{SL}(n, \mathbb{R}) / \mathrm{SO}(n)$. These metrics are all of the Berwald type.

Next we consider nonsymmetric polar homogeneous manifolds. By Kollross and Podestà [2003] classified the polar homogeneous spaces of a connected, simply connected, simple Lie group. Combining their list and Theorem 4.1 gives this:

Theorem 4.5. Let $G / H$ be a connected, simply connected, isotropic polar homogeneous manifold, where $G$ is a simply connected simple compact Lie group and $H$ is a closed subgroup of $G$. Then the pair $(G, H)$ must be either a Riemannian symmetric pair or one of the pairs in Table 1. Among the manifolds in Table 1, any $G$-invariant Finsler metric on $G / H$ must be Riemannian in types VIII and IX. In any of the other types, however, there exist infinitely many $G$-invariant nonRiemannian Finsler metrics on $\mathrm{G} / \mathrm{H}$.

Proof. Because Kollross and Podestà [2003] listed the isotropic polar homogeneous manifolds, we only need to find which type is of rank 1 . Since $H$ is compact, the action of $H$ on the tangent space $T_{o}(G / H)$ leaves the inner product invariant. Therefore, we can view $H$ as a subgroup of $\mathrm{SO}(m)$ (note that $H$ is connected in all the types in Table 1), where $m=\operatorname{dim} T_{o}(G / H)$. Therefore, to find out which type is of rank 1, we only need to find in which case $H$ acts transitively on $S^{m-1}$. The compact connected subgroups of $\mathrm{SO}(m)$ that act transitively on $S^{m-1}$ were classified by Montgomery, Samelson, and Borel [Besse 1987]. Therefore the 


\begin{tabular}{cccc}
\hline Type & $G$ & $H$ & Rank \\
\hline I & $\mathrm{SU}(n+1)$ & $\mathrm{SU}(n)$ & $\geq 2$ \\
II & $\mathrm{Sp}(n+1)$ & $\mathrm{Sp}(n)$ & $\geq 2$ \\
III & $\mathrm{Sp}(n+1)$ & $\mathrm{U}(1) \times \mathrm{Sp}(n)$ & $\geq 2$ \\
IV & $\mathrm{SU}(p+q)$ & $\mathrm{SU}(p) \times \mathrm{SU}(q), p<q$ & $\geq 2$ \\
V & $\mathrm{Spin}(2 n)$ & $\mathrm{SU}(n), n$ odd & $\geq 2$ \\
VI & $\mathrm{E}_{6}$ & $\mathrm{Spin}(10)$ & $\geq 2$ \\
VII & $\mathrm{Spin}(9)$ & $\mathrm{Spin}(7)$ & $\geq 2$ \\
VIII & $\mathrm{Spin}(7)$ & $\mathrm{G}_{2}$ & 1 \\
IX & $\mathrm{G}_{2}$ & $\mathrm{SU}(3)$ & 1 \\
\hline
\end{tabular}

Table 1. Pairs $(G, H)$ occurring in Theorem 4.5.

theorem can be proved through a case-by-case computation of the dimensions of the manifolds $G / H$.

Now we give a description of the invariant Finsler metrics on the homogeneous manifolds of type I in Table 1.

Example 4.6. Consider a homogeneous manifold

$$
M=G / H=\mathrm{SU}(n+1) / \mathrm{SU}(n), \quad \text { with } n \geq 2 .
$$

Now we give a realization of the manifold $M$. The group $G=\mathrm{SU}(n+1)$ acts in the standard way on the standard Hermitian space $\mathbb{C}^{n+1}=\mathbb{R}^{2 n+2}$. The action keeps the sphere $S^{2 n+1}$ invariant, and the restriction of the action to the sphere is transitive. The subgroup $H=\mathrm{SU}(n)$ can be identified with the isotropic subgroup of $G$ at the point $o=(1,0, \ldots, 0)^{\prime} \in S^{2 n+1}$, that is,

$$
A \hookrightarrow\left(\begin{array}{ll}
1 & 0 \\
0 & A
\end{array}\right) \text { for } A \in H .
$$

Therefore $M$ is just the sphere $S^{2 n+1}$. Now we make some observations on the isotropic representation. By selecting a certain local coordinate system, we can identify the tangent space of $G / H$ at $o$ with the hyperplane

$$
P=\left\{\left(1, b_{1}, b_{2}, \ldots, b_{2 n+1}\right)^{\prime} \in \mathbb{R}^{2 n+1}\right\}
$$

through the mapping

$$
b=\left(b_{1}, b_{2}, \ldots, b_{2 n+1}\right) \hookrightarrow\left(1, b_{1}, b_{2}, \ldots, b_{2 n+1}\right)^{\prime} .
$$

Then the isotropic representation can be described as follows. For

$$
b=\left(b_{1}, b_{2}, \ldots, b_{2 n+1}\right) \in T_{o}(G / H) \text { and } A \in H \text {, }
$$


define $\tilde{b}=\left(1+b_{1} \sqrt{-1}, b_{2}+b_{3} \sqrt{-1}, \ldots, b_{2 n}+b_{2 n+1} \sqrt{-1}\right)^{\prime} \in \mathbb{C}^{n+1}$. Let

$$
c=\left(\begin{array}{ll}
1 & 0 \\
0 & A
\end{array}\right) \cdot \tilde{b}=\left(1+b_{1} \sqrt{-1}, c_{1}+c_{2} \sqrt{-1}, \ldots, c_{2 n-1}+c_{2 n} \sqrt{-1}\right)^{\prime} \in \mathbb{C}^{n+1},
$$

where $c_{j}, j=1,2, \ldots, 2 n$ are real numbers. Then

$$
A \cdot b=\left(b_{1}, c_{1}, c_{2}, \ldots, c_{2 n}\right) .
$$

Therefore, the isotropic representation is equal to the identity transformation on the subspace $V_{1}=\left\{\left(b_{1}, 0, \ldots, 0\right) \in T_{o}(M)\right\}$. On the other hand, the action of $H$ on the subspace $\left.V_{2}=\left\{\left(0, b_{2}, \ldots, b_{2 n+1}\right) \in T_{o}(M)\right)\right\}$ is just the standard action of the group $\mathrm{SU}(n)$ on $\mathbb{C}^{n}$. Therefore, $H$ is transitive on the unit sphere in $V_{2}$. From this, we see that the action of $H$ on $V_{2}$ is polar of rank 1 . Since $T_{o}(M)$ is the orthogonal sum of $V_{1}$ and $V_{2}$, the action of $H$ on $T_{o}(M)$ is polar, and a Cartan space can be chosen to be $\mathfrak{a}=V_{1}+\mathfrak{a}_{2}$, where $\mathfrak{a}_{2}$ is an arbitrary one-dimensional subspace in $V_{2}$. As in Example 4.3 any Minkowski norm $F$ on a satisfying

$$
F( \pm x \pm y)=F(x+y), \quad x \in V_{1}, y \in \mathfrak{a}_{2}
$$

can be extended uniquely to a $H$-invariant Minkowski norm on $T_{o}(M)$, and hence corresponds to a $G$-invariant Finsler metric on $M$.

\section{General geometric properties}

Let $(G, K)$ be a Riemannian symmetric pair. Then by the results of [Szabó 1981] it is easily seen that any $G$-invariant Finsler metric on $G / K$ must be a (reversible or nonreversible) affine symmetric Berwald space. By Dadok's results, we have seen that a polar representation must have the same orbits as the isotropic representation of a certain Riemannian symmetric space. It is therefore natural to ask whether the result above holds for polar homogeneous spaces, that is, whether any invariant Finsler metric on a polar homogeneous space must be Berwaldian. We will investigate this problem in this section. It is somehow surprising that the answer is negative. In fact we can prove that in any polar homogeneous manifold in Table 1, an invariant Finsler metric $F$ on $G / H$ is Berwaldian if and only if it is Riemannian. As an application, we show that on any polar homogeneous space of rank at least 2 in Table 1, there exist infinitely many invariant Finsler metrics that are reversible, non-Berwaldian and of vanishing S-curvature. The problem of the existence of such spaces was posed by Shen [2009], and in our paper [Deng and Hou $\geq 2010$ ], we constructed some low-dimensional examples of Finsler spaces with the above properties.

We begin with the notions of weakly symmetric Finsler spaces and geodesic orbit Finsler spaces. Let $(M, F)$ be a connected Finsler space. Then $(M, F)$ is called weakly symmetric if for any two points $p$ and $q$ there exists an isometry $\sigma$ 
of $(M, F)$ that interchanges them, that is, $\sigma(p)=q$ and $\sigma(q)=p$. It is called a geodesic orbit Finsler space if any geodesic $\gamma$ is the orbit of a one-parameter subgroup of the full group of isometries, that is, if there exists a vector $X$ in the Lie algebra $\mathfrak{g}$ of the full group $G$ of isometries such that $\gamma(t)=\exp (t X) \cdot o$, where $o=\gamma(0)$ and exp is the exponential mapping of $G$. It is obvious that a weakly symmetric space must be reversible and homogeneous and that a geodesic orbit Finsler space must be homogeneous. Berndt et al. [1997] proved that a connected weakly symmetric Riemannian manifold must be a geodesic orbit space. Their proof is also valid for the Finslerian case, so a weakly symmetric Finsler must be a geodesic orbit space. Also it is easy to prove that a geodesic orbit space must have vanishing S-curvature [Deng and $\mathrm{Hou} \geq 2010$ ].

Nguyen [2000] introduced a way to construct weakly symmetric Riemannian manifolds. Let $G$ be a connected Lie group and $\theta$ be an involutive automorphism of $G$. Suppose $H$ is a $\theta$-stable compact subgroup of $G$. Select a complement subspace $\mathfrak{m}$ of $\mathfrak{h}$ in $\mathfrak{g}$ that is also invariant under $\operatorname{Ad}_{\mathfrak{g} / \mathfrak{h}}(H)$. Then $(G, H, \theta)$ is called a weakly symmetric triple if, given any element $X \in \mathfrak{m}$, there exists an element $h \in H$ such that $(\operatorname{Ad}(h)) \circ d \theta(X)=-X$. Nguyen proved that if $(G, H, \theta)$ is weakly symmetric pair, then any $G$-invariant Riemannian metric on $G / H$ is weakly symmetric. Using this method we can also construct weakly symmetric Finsler spaces.

Proposition 5.1. If $(G, H, \theta)$ is a weakly symmetric triple, then any $G$-invariant reversible Finsler metric on $G / H$ is weakly symmetric.

The proof is similar to the Riemannian case, so we omit it [Nguyen 2000].

Theorem 5.2. Let $G / H$ be one of the polar homogeneous spaces in Table 1 (with nontrivial subgroup $H$ ) that is not of type II (that is, not $\operatorname{Sp}(n+1) / \operatorname{Sp}(n)$ ). Then any reversible $G$-invariant Finsler metric on $G / H$ must be weakly symmetric. In the coset space $\operatorname{Sp}(n) / \operatorname{Sp}(n-1)$ for $n \geq 2$, there exist infinitely many invariant weakly symmetric non-Riemannian Finsler metrics. In particular, in any of the polar homogeneous manifolds of rank at least 2 in Table 1, there exist infinitely many invariant weakly symmetric non-Riemannian Finsler metrics.

Proof. The first claim follows from the classification of compact weakly symmetric Riemannian spaces by Nguyen [2000] and Yakimova [2004]. Also some of these homogeneous manifolds are known to be weakly symmetric [Ziller 1996]. We now give a case-by-case clarification. The manifolds $\mathrm{SU}(n) / \mathrm{SU}(n-1)$, with $n \geq 3$, are known to be weakly symmetric [Ziller 1996]. The involutive automorphism $\theta$ of $\mathrm{SU}(n)$ can be defined in the following way: Let $\mathrm{SU}(n)$ act in the standard way on the unit sphere in $\mathbb{C}^{n}$, and let $\mu$ be the transformation taking the complex conjugation on each coordinate. Define $\theta(g)=\mu g \mu^{-1}$. It is easy to check that $(\mathrm{SU}(n), \mathrm{SU}(n-1), \theta)$ is a weakly symmetric triple. Therefore by Proposition 5.1, 
$\mathrm{SU}(n) / \mathrm{SU}(n-1)$ endowed with any invariant reversible non-Riemannian Finsler metric must be a weakly symmetric Finsler space. This argument is also valid for the homogeneous space $\mathrm{SU}(p+q) / \mathrm{SU}(p) \times \mathrm{SU}(q)$, with $p<q$. Therefore $\mathrm{SU}(p+q) / \mathrm{SU}(p) \times \mathrm{SU}(q)$ endowed with any reversible Finsler spaces must be weakly symmetric. According to Nguyen [2000], the homogeneous space $G / H=$ $\mathrm{Sp}(n) / \mathrm{Sp}(n-1) \cdot U(1)$ is weakly symmetric with respect to $G$, meaning that for any $X \in T_{o}(G / H)$, there exists an $h \in H$ such that $d h(X)=-X$. Similarly to the Riemannian case, this means that any invariant reversible Finsler metric on $G / H$ must be weakly symmetric [Ziller 1996]. Now by [Nguyen 2000], the space $\mathrm{SO}(2 n) / \mathrm{SU}(n)$, with $n$ odd, is weakly symmetric. Since the space $\operatorname{Spin}(2 n) / \mathrm{SU}(n)$ is the universal covering of $\mathrm{SO}(2 n) / \mathrm{SU}(n)$, we see that $\operatorname{Spin}(2 n) / \mathrm{SU}(n)$ endowed with any reversible must weakly symmetric [Yakimova 2004]. The situation is the same for the space $\operatorname{Spin}(9) / \operatorname{Spin}(7)$. Finally, the space $E_{6} / \operatorname{Spin}(10)$ appears in the list in [Nguyen 2000] (note that $D_{5}$ is exactly $\operatorname{Spin}(10)$ ). Therefore, if $G / H$ is a homogeneous manifold of rank at least 2 in Table 1 not of type II, then any invariant reversible Finsler metric on $G / H$ must be weakly symmetric.

Now we consider spaces of type II, that is, $\operatorname{Sp}(n) / \operatorname{Sp}(n-1)$ with $n \geq 2$. Note that $\operatorname{Sp}(n)$ acts transitively on the sphere $S^{4 n-1}$ in the standard way, and that any isotropy subgroup is isomorphic to $\operatorname{Sp}(n-1)$. Hence $\operatorname{Sp}(n) / \operatorname{Sp}(n-1)=S^{4 n-1}$. In this way, we can also view $\mathrm{Sp}(n)$ as a subgroup of $\mathrm{SU}(2 n)$. Since we have $\mathrm{SU}(2 n) / \mathrm{SU}(2 n-1)=S^{4 n-1}$, we see that any $\mathrm{SU}(2 n)$ invariant Finsler metric on the sphere $S^{4 n-1}$ must be an invariant metric on the coset space $\operatorname{Sp}(n) / \operatorname{Sp}(n-1)$. From this the theorem follows.

Theorem 5.3. Let $G$ be a connected simply connected compact simple Lie group and $H$ be a closed subgroup of $G$. Let $G / H$ be a nonsymmetric polar homogeneous manifold. Then a $G$-invariant Finsler metric on $G / H$ is Berwaldian if and only if it is Riemannian.

Lemma 5.4. Let $G$ be a compact connected Lie group and $H$ be a closed subgroup of $G$. Suppose $G / H$ is diffeomorphic to the $n$-sphere, with $n \geq 2$. Then any $G$ invariant Riemannian metric on $G / H$ is holonomy irreducible.

Proof. Suppose conversely that a $G$-invariant Riemannian metric $Q$ on $G / H$ is holonomy reducible. Then by the de Rham decomposition theorem, we have a Riemannian manifold decomposition

$$
G / H=M_{1} \times M_{2} \times \cdots \times M_{s},
$$

where $M_{1}, M_{2}, \ldots, M_{s}$ are holonomy irreducible. By Hano's result [1955], the full group $K$ of isometries of $(G / H, Q)$ is isomorphic to the product of the groups of isometries of $M_{i}$ for $1 \leq i \leq s$. This fact combined with the homogeneity of $(G / H, Q)$ implies that for each $i$, the group of isometries of $M_{i}$ must be transitive 
on $M_{i}$; in particular, it has dimension at least 1 . Thus the identity component $K^{0}$ of $K$ is not a simple Lie group. Now by the complete list of compact connected Lie groups that act transitively on the spheres [Besse 1987], $K^{0}$ must be one of $\mathrm{U}(n)$ with $n \geq 2, \operatorname{Sp}(n) \operatorname{Sp}(1)$ with $n \geq 1$, or $\operatorname{Sp}(n) \mathrm{U}(1)$ with $n \geq 1$. This means that $s=2$ and $M_{1}$ can be chosen to be a coset space of $\mathrm{U}(1)=S^{1}$ or $\mathrm{Sp}(1)=\mathrm{SU}(2)=S^{3}$. But it is easily seen that the coset spaces of $\mathrm{U}(1)$ or $\mathrm{Sp}(1)$ must be diffeomorphic to $S^{1}, S^{2}$ or $S^{3}$. Therefore we have $S^{n}=G / H=S^{j} \times M_{2}$ for $n>j$. But this decomposition is impossible since the $j$-th homotopy group of $S^{n}$ (where $j=1,2$, or 3 and $n>j)$ is the identity group and $\pi_{j}\left(S^{j}\right)=\mathbb{Z}$.

Oniscik [1963] proved that the only compact connected groups that act transitively on the projective complex spaces are $\mathrm{SU}(n)$ (on $\mathbb{C} P^{n-1}$ ), and $\operatorname{Sp}(n)$ (on $\left.\mathbb{C} P^{2 n-1}\right)$. Similar to Lemma 5.4, we have this:

Lemma 5.5. Let $G$ be a compact connected Lie group and $H$ be a closed subgroup of $G$. Suppose the coset space $G / H$ is diffeomorphic to the projective complex space $\mathbb{C} P^{n}$. Then any $G$-invariant Riemannian metric on $G / H$ must be holonomy irreducible.

To state the next lemma, we need some definitions about Hermitian symmetric spaces. Let $(G, H)$ be an irreducible Riemannian metric of compact or noncompact type. Then it is well known that $G / H$ can be made into a Hermitian symmetric space if and only if the (connected) compact subgroup $H$ has nondiscrete center, in which case the center of $H$ is a one-dimensional Lie group [Helgason 1978]. Let $\mathfrak{g}=\mathfrak{h}+\mathfrak{m}$ be the canonical decomposition of the Lie algebra. Let $\mathfrak{a}$ be a maximal abelian subspace of $\mathfrak{m}$ and extend $\mathfrak{a}$ to a Cartan subalgebra $\mathfrak{t}$ of $\mathfrak{g}$. Then on the subspace $\mathfrak{m}$ there is a complex structure $J$ corresponding to the root system of $(\mathfrak{g}, \mathfrak{t})$ (not necessary the complex structure induced by that of the manifold $G / H)$ [Korányi and Wolf 1965]. Let $Z^{J}$ be the element in the center $\mathfrak{z} \mathfrak{h}$ of $\mathfrak{h}$ that corresponds to the complex structure above. Then we have a decomposition $Z^{J}=Z^{0}+Z^{\prime}$, where $Z^{0}$ defines the complex structure on the polydisc or the polysphere inside $G / H$ when $G / H$ is realized as a generalized half-plane [Korányi and Wolf 1965] and $Z^{\prime}$ is an element in $\mathfrak{h}$ that centralizes $\mathfrak{a}$. The Hermitian symmetric space $G / H$ is said to be of tube type if in the decomposition above we have $Z^{\prime}=0$. Let $H=Z_{H} \cdot H_{S}$ be the decomposition of $H$, where $Z_{H}$ is the one-dimensional center of $H$ and $H_{s}$ is the semisimple part of $H$. Let $S^{\prime}=\left\{\exp \left(t Z^{\prime}\right) \mid t \in \mathbb{R}\right\}$. It is known that if $G / H$ is not of the tube type, then $Z^{\prime}$ is not in the center $\mathfrak{z}_{\mathfrak{h}}$ and we have $H=S^{\prime} H_{s}=H_{s} S^{\prime}$. Further,

$$
\mathfrak{m}=\operatorname{Ad}\left(H_{S}\right)(\mathfrak{a}) .
$$

Lemma 5.6. Let $G$ be a compact connected simply connected simple Lie group and $H$ be a closed subgroup of $G$ such that $(G, H)$ is an irreducible Hermitian 
symmetric pair of nontube type. Let $H=Z_{H} H_{S}$ be the decomposition of $H$, where $Z_{H}$ is the one-dimensional center of $H$ and $H_{S}$ is the semisimple part of $H$. Then any $G$-invariant Riemannian metric on the coset space $G / H_{s}$ must be holonomy irreducible.

Proof. Let $\mathfrak{g}=\mathfrak{h}+\mathfrak{m}$ be the canonical decomposition of the symmetric pair $(G, H)$. Then we can identify the tangent space $T_{o}(G / H)$ with $\mathfrak{m}$. Since $(G, H)$ is irreducible, the action of $H$ on $\mathfrak{m}$ is irreducible. Now we claim that the action of the semisimple part $H_{s}$ of $H$ on $\mathfrak{m}$ is also irreducible. In fact, if this is not true, then we can find a nontrivial subspace $V_{1}$ of $\mathfrak{m}$ that is invariant under $H_{s}$. Let $X \in V_{1}$. Then by (5-1) there exists $k \in H_{s}$ and $X_{\mathfrak{a}} \in \mathfrak{a}$ such that $X=\operatorname{Ad}(k)\left(X_{\mathfrak{a}}\right)$. Now for any $s \in S^{\prime}$, select $s_{1} \in S^{\prime}, k_{1} \in H_{s}$ such that $s k=k_{1} s_{1}$. Then

$$
\begin{aligned}
\operatorname{Ad}(s)(X) & =\operatorname{Ad}(s) \operatorname{Ad}(k)\left(X_{\mathfrak{a}}\right)=\operatorname{Ad}\left(k_{1} s_{1}\right)\left(X_{\mathfrak{a}}\right)=\operatorname{Ad}\left(k_{1}\right)\left(X_{\mathfrak{a}}\right) \\
& =\operatorname{Ad}\left(k_{1} k^{-1}\right) \operatorname{Ad}(k)\left(X_{\mathfrak{a}}\right)=\operatorname{Ad}\left(k_{1} k^{-1}\right)(X) \in V_{1},
\end{aligned}
$$

where we have used the fact that $Z^{\prime}$ centralizes $\mathfrak{a}$. Now (5-2) means that $V_{1}$ is also invariant under the action of $S^{\prime}$. Hence it is invariant under $H$. This contradicts the assumption that $G / H$ is irreducible, and proves our claim. The claim means that the action of $H_{s}$ on the tangent space $T_{o}\left(G / H_{S}\right)$, which can be identified with $\mathfrak{s}+\mathfrak{m}$ (direct sum), where $\mathfrak{s}$ is the one-dimensional Lie algebra of $S^{1}$, decomposes as the sum of irreducible subspaces $\mathfrak{s}$ and $\mathfrak{m}$. By Schur's lemma, this implies that any $H_{s}$-invariant inner product on $T_{o}\left(G / H^{\prime}\right)$ must be of the form

$$
Q_{1}+\left.\alpha(-B)\right|_{\mathfrak{m} \times \mathfrak{m}},
$$

where $Q_{1}$ is an arbitrary inner product on $\mathfrak{a}$ (which is unique up to a positive scalar), $B$ is the Cartan-Killing form of the Lie algebra $\mathfrak{g}$, and $\alpha$ is an arbitrary positive number. Now by the construction of D'Atri and Ziller [1979], any inner product of the form (5-3) induces a naturally reductive $G$-invariant Riemannian metric on $G / H^{\prime}$. Such a Riemannian metric on a simply connected coset space of a connected simple Lie group must be holonomy irreducible [Kobayashi and Nomizu 1969, page 215].

Proof of Theorem 5.3. We need only prove the "only if" part. We divide the homogeneous manifolds in Table 1 into three groups. Group 1 consists of types I, II, VII, VIII, and IX. Homogeneous manifolds in this group are diffeomorphic to a sphere. Group 2 consists of type III, the manifold $\mathrm{Sp}(n) /(\mathrm{U}(1) \times \mathrm{Sp}(n-1))$. It has a symmetric extension $((\mathrm{SU}(2 n), \mathrm{SU}(2 n-1) \times \mathrm{U}(1))$; in other words, the quotient $\mathrm{Sp}(n) /(\mathrm{U}(1) \times \mathrm{Sp}(n-1))$ is diffeomorphic to the projective complex space

$$
\mathbb{C} P^{2 n-1}=\mathrm{SU}(2 n) /(\mathrm{SU}(2 n-1) \times \mathrm{U}(1)),
$$


where we consider $\mathrm{Sp}(n)$ as a subgroup of $\mathrm{SU}(2 n)$. Group 3 consists of types IV, $\mathrm{V}$, and VI. Homogeneous manifolds in this type are $S^{1}$-bundles over Hermitian symmetric spaces of nontube type [Nguyen 2000]. Let $\mathrm{G} / \mathrm{H}$ be one of the homogeneous manifolds in Table 1. Then by Lemmas 5.4, 5.5, and 5.6, we have seen that any $G$-invariant Riemannian metric on $G / H$ must be holonomy irreducible. Suppose $F$ is an invariant Finsler metric on $G / H$ of the Berwald type. Then there exists a Riemannian metric $Q$ on $G / H$ whose Levi-Civita connection coincides with the Chern connection of $F$ [Szabó 1981]. Let $A(Q)$ and $I(Q)$ be the group of affine transformations and the group of isometries of $Q$. Then any isometry of $F$ must be contained in $A(Q)$ [Deng and Hou 2005b]. In particular, $G \subset A(Q)$. On the other hand, since $G / H$ is compact, we have $A(Q)^{0}=I(Q)^{0}$ [Kobayashi and Nomizu 1963, page 244]. Since $G$ is connected, we have $G \subset A(Q)^{0}=I(Q)^{0}$. That is, any element of $G$ is an isometry of $Q$, or in other words, $Q$ is a $G$ invariant Riemannian metric and hence must be holonomy irreducible. If $F$ is not Riemannian, then according to [Szabó 1981], $(G / H, Q)$ must be an irreducible Riemannian symmetric space of rank at least 2. In particular, let $K$ be the full group of isometries of $Q$, let $K_{0}$ be the identity component of $K$, and let $N$ be the isotropic subgroup of $K_{0}$ at a fixed point. Then $G \subset K^{0}$, and $\left(K_{0}, N\right)$ is a Riemannian symmetric pair [Helgason 1978]. This means that the pair $(G, H)$ has a symmetric extension with rank at least 2 . The symmetric extension of weakly symmetric homogeneous manifolds has been classified by Yakimova [2004]. The list shows that the manifolds of types I, III, VII, VIII, and IX in Table 1 have symmetric extension of rank 1 , and that the manifolds in other types do not admit any symmetric extension. On the other hand, $\operatorname{Sp}(n) / \operatorname{Sp}(n-1)$ is the only homogeneous manifold in Table 1 that is not weakly symmetric. But $\operatorname{Sp}(n) / \operatorname{Sp}(n-1)$ is diffeomorphic to the sphere $S^{4 n-1}$. Hence $(\operatorname{Sp}(n), \operatorname{Sp}(n-1))$ has as its only symmetric extension $(\mathrm{SO}(4 n), \mathrm{SO}(4 n-1))$, which is of rank 1 . This contradiction proves the theorem.

Since a weakly symmetric Finsler space has vanishing S-curvature, combining Theorems 5.2 and 5.3 gives this corollary:

Corollary 5.7. Let $G$ be a connected simply connected compact Lie group, and let $H$ be a closed subgroup of $G$. Let $G / H$ be a nonsymmetric polar homogeneous manifold of rank 2. Then there exist infinitely many invariant Finsler metrics on $G / H$ that are reversible, non-Berwaldian, and of vanishing $S$-curvature.

\section{Randers metrics}

We now consider invariant Randers metrics on the homogeneous manifolds in Table 1, and give a (global) complete classification of such metrics. As pointed 
out in Theorem 5.3, all the non-Riemannian Randers metrics we find are nonBerwaldian. As an application, we give a classification of homogeneous Randers spaces with positive constant flag curvature.

We first recall some known results. Let $G$ be a Lie group, and let $H$ be a closed subgroup of $G$. Suppose $Q$ is an invariant Riemannian metric on $G / H$. Then by the results of [Deng and Hou 2004b], there exists a bijection between the invariant Randers metrics on $G / H$ with underlying Riemannian metric $Q$ and the invariant vector fields on $G / H$ with length $<1$. Suppose the coset space $G / H$ is reductive, that is, that there exists a subspace $\mathfrak{m}$ of $\mathfrak{g}$ (the Lie algebra of $G$ ) such that

$$
\mathfrak{g}=\mathfrak{h}+\mathfrak{m} \quad \text { (direct sum of subspaces), }
$$

and $\operatorname{Ad}(h) \mathfrak{m} \subset \mathfrak{m}$, for all $h \in H$. Then invariant vector fields are in bijection with the set

$$
V=\{X \in \mathfrak{m} \mid \operatorname{Ad}(h)(X)=X \text { for all } h \in H\} .
$$

Therefore, to find all the invariant Randers metrics on $G / H$, we need first to find all the invariant Riemannian metrics with respect to which all vectors in $V$ have length less than 1.

Now we consider $\mathrm{SU}(n) / \mathrm{SU}(n-1)$. In this case we have a decomposition

$$
\mathfrak{s} u(n)=\mathfrak{s} u(n-1)+\mathfrak{m},
$$

where

$$
\mathfrak{m}=\left\{\left(\begin{array}{cc}
a \sqrt{-1} & \alpha \\
-\bar{\alpha}^{\prime} & -\frac{1}{n-1} a \sqrt{-1} I_{n-1}
\end{array}\right) \mid a \in \mathbb{R}, \alpha \in \mathbb{C}^{n-1}\right\} .
$$

A direct computation shows that

$$
V=\left\{\operatorname{diag}\left(-a \sqrt{-1}, \frac{1}{n-1} a \sqrt{-1}, \ldots, \frac{1}{n-1} a \sqrt{-1}\right) \mid a \in \mathbb{R}\right\} .
$$

Theorem 6.1. There is a one-to-one correspondence between the invariant Randers metrics on $\mathrm{SU}(n) / \mathrm{SU}(n-1)$ and the Minkowski norms on $\mathfrak{m}$ in (6-2). This bijection is defined by

$$
F_{o}(X)=\sqrt{c_{1} a^{2}+c_{2} \alpha \bar{\alpha}^{\prime}}+c_{1} c a, \quad X=\left(\begin{array}{cc}
a \sqrt{-1} & \alpha \\
-\bar{\alpha}^{\prime} & -\frac{1}{n-1} a \sqrt{-1} I_{n-1}
\end{array}\right) \in \mathfrak{m},
$$

where $c_{1}, c_{2}$ are positive real parameters and $c$ is a real number with $|c|<1 / \sqrt{c_{1}}$.

Among these Randers metrics, given any positive number $k$, the family with parameters

$$
c_{1}=\frac{d^{2}+k^{2}-k d^{2}}{\left(k-d^{2}\right)^{2}}, \quad c_{2}=\left(\frac{k}{k-d^{2}}\right)^{2}, \quad c=\frac{d\left(d^{2}-k\right)}{d^{2}+k^{2}-k d^{2}},
$$


has constant flag curvature $k$, where $d$ is a real parameter satisfying $|d|<\sqrt{k}$. Moreover, up to isometry these are all the connected simply connected homogeneous Randers spaces with positive constant flag curvature.

Moreover, any non-Riemannian Randers metric above is not of the Douglas type, and hence is not projectively flat.

Proof. As stated above, to obtain the classification of invariant Randers metrics on $\mathrm{SU}(n) / \mathrm{SU}(n-1)$, we first need to determine all invariant Riemannian metrics thereon. These metrics are in bijection with the $\mathrm{SU}(n-1)$-invariant inner products on $\mathfrak{m}$. Now the vector space $\mathfrak{m}$, as a representation space of $\operatorname{SU}(n-1)$, has the decomposition $\mathfrak{m}=V+\mathfrak{m}_{1}$, where

$$
\mathfrak{m}_{1}=\left\{\left(\begin{array}{cc}
0 & \alpha \\
-\bar{\alpha}^{\prime} & 0
\end{array}\right) \mid \alpha \in \mathbb{C}^{n-1}\right\} .
$$

Moreover, the subrepresentations $V$ and $\mathfrak{m}_{1}$ are irreducible. By Schur's lemma, it is easily seen that any $\mathrm{SU}(n-1)$-invariant inner product on $\mathfrak{m}$ must be of the form

$$
\left\langle X_{1}, X_{2}\right\rangle=c_{1} a_{1} a_{2}+c_{2} \operatorname{Re}\left(\alpha_{1} \bar{\alpha}_{2}^{\prime}\right) \text { for } c_{1}, c_{2}>0 \text {, }
$$

where

$$
X_{i}=\left(\begin{array}{cc}
a_{i} \sqrt{-1} & \alpha_{i} \\
-\bar{\alpha}_{i}^{\prime} & -\frac{1}{n-1} a_{i} \sqrt{-1}
\end{array}\right) \quad \text { for } i=1,2 .
$$

Therefore, the $\mathrm{SU}(n)$-invariant Randers metric on $\mathrm{SU}(n) / \mathrm{SU}(n-1)$ determined by $\langle\cdot, \cdot\rangle$ and

$$
X_{0}=\left(\begin{array}{cc}
c \sqrt{-1} & 0 \\
0 & -\frac{1}{n-1} c \sqrt{-1}
\end{array}\right) \in V, \quad \text { with } \sqrt{c_{1}} c<1,
$$

must be the one stated in the theorem.

Now we prove the theorem's second claim. Note that $\mathrm{SU}(n)$ is a closed subgroup of $\mathrm{SO}(2 n)$ and is transitive on the sphere $S^{2 n-1}=\mathrm{SO}(2 n) / \mathrm{SO}(2 n-1)$. This means that any $\mathrm{SO}(2 n)$-invariant Riemannian metric must be $\mathrm{SU}(n)$-invariant. Thus for any $k>0$ there is one $\mathrm{SU}(n)$-invariant Riemannian metric on $\mathrm{SU}(n) / \mathrm{SU}(n-1)$ with constant sectional curvature $k$. We denote this Riemannian metric by $Q_{k}$, and next determine it explicitly. For two orthogonal unit vectors $X, Y \in \mathfrak{m}$, the sectional curvature of the plane spanned by $X, Y$ is given by [Besse 1987, p. 183]

$$
\begin{aligned}
K(X, Y)=-\frac{3}{4}\left|[X, Y]_{\mathfrak{m}}\right|^{2}-\frac{1}{2}\left\langle[X,[X, Y]]_{\mathfrak{m}}, Y\right\rangle \\
-\frac{1}{2}\left\langle[Y,[Y, X]]_{\mathfrak{m}}, X\right\rangle+|U(X, Y)|^{2}-\langle U(X, X), U(Y, Y)\rangle,
\end{aligned}
$$

where $\langle\cdot, \cdot\rangle$ is the inner product determined by $Q_{k},|\cdot|$ is the length function of $\langle\cdot, \cdot\rangle,[\cdot, \cdot]$ is the Lie bracket of $\mathfrak{s u}(n),[X, Y]_{\mathfrak{m}}$ denotes the projection of 
$[X, Y]$ to $\mathfrak{m}$ corresponding to the decomposition (6-1), and $U$ is a symmetric bilinear mapping from $\mathfrak{m} \times \mathfrak{m}$ to $\mathfrak{m}$ defined by

$$
\langle U(X, Y), Z\rangle=\frac{1}{2}\left(\left\langle[Z, X]_{\mathfrak{m}}, Y\right\rangle+\left\langle[Z, Y]_{\mathfrak{m}}, X\right\rangle\right) .
$$

Up to homotheties, the set of invariant Riemannian metrics on $\mathrm{SU}(n) / \mathrm{SU}(n-1)$ has dimension 1 , so there must be a $c_{1}>0$ such that the inner product $c_{1} a_{1} a_{2}+\operatorname{Re}\left(\alpha_{1} \bar{\alpha}_{2}^{\prime}\right)$ defines a Riemannian metric with constant sectional curvature. To find this $c_{1}$, we select three vectors in $\mathfrak{m}$ :

$$
\begin{aligned}
& X_{1}=\operatorname{diag}\left(\frac{\sqrt{-1}}{\sqrt{c_{1}}},-\frac{\sqrt{-1}}{(n-1) \sqrt{c_{1}}} I_{n-1}\right), \\
& X_{2}=\left(\begin{array}{cc}
0 & \alpha_{2} \\
-\alpha_{2}^{\prime} & 0
\end{array}\right), \quad \alpha_{2}=(1,0, \ldots, 0) \in \mathbb{R}^{n-1}, \quad X_{3}=\sqrt{-1} X_{2} .
\end{aligned}
$$

Then a direct (albeit somewhat tedious) computation shows that

$$
\begin{aligned}
& U\left(X_{1}, X_{1}\right)=U\left(X_{2}, X_{2}\right)=U\left(X_{3}, X_{3}\right)=0, \\
& U\left(X_{1}, X_{2}\right)=\frac{1}{2}\left(\frac{n}{(n-1) \sqrt{c_{1}}}-2 \sqrt{c_{1}}\right) X_{3}, \\
& U\left(X_{1}, X_{3}\right)=\frac{1}{2}\left(-\frac{n}{(n-1) \sqrt{c_{1}}}+2 \sqrt{c_{1}}\right) X_{2}, \\
& U\left(X_{2}, X_{3}\right)=0 .
\end{aligned}
$$

Substituting the above into the formula of sectional curvature, we get

$$
K\left(X_{1}, X_{2}\right)=c_{1}+\frac{n}{n-1}\left(1-\sqrt{c_{1}}\right) \quad \text { and } \quad K\left(X_{2}, X_{3}\right)=4-3 c_{1} .
$$

Now the equation $K\left(X_{1}, X_{2}\right)=K\left(X_{2}, X_{3}\right)$ has a unique positive solution $c_{1}=1$, and in this case the sectional curvature is equal to 1 . Therefore the inner product

$$
(1 / k) a_{1} a_{2}+(1 / k) \operatorname{Re}\left(\alpha_{1} \bar{\alpha}_{2}^{\prime}\right)
$$

defines the invariant Riemannian metric $Q_{k}$ on $\mathrm{SU}(n) / \mathrm{SU}(n-1)$ with constant sectional curvature $k$.

Now for any $X \in V$ with $Q_{k}(X, X)<1$, we can construct an invariant Randers metric $F_{k, X}$ on $\mathrm{SU}(n) / \mathrm{SU}(n-1)$. Since $Q_{k}$ is $\mathrm{SU}(n)$-invariant, the one parameter group $\{\exp (t X) \mid t \in \mathbb{R}\}$ consists of isometries of $Q_{k}$. In particular, for any $U, V \in \mathfrak{m}$, we have $Q_{k}(\operatorname{Ad}(\exp (t X))(U), \operatorname{Ad}(\exp (t X))(V))=Q_{k}(U, V)$ for all $t$. Taking the derivative and considering the value at $t=0$, we get

$$
Q_{k}\left([X, U]_{\mathfrak{m}}\right)+Q_{k}\left(U,[X, V]_{\mathfrak{m}}\right)=0 .
$$

In other words, $\mathscr{L}_{\tilde{X}} Q_{k}$ is equal to 0 at the origin. Since both the Riemannian metric and the vector field $\tilde{X}$ are $\operatorname{SU}(n)$-invariant, we have $\mathscr{L}_{\tilde{X}} Q_{k}=0$ everywhere. By 
the criterion for a Randers metric to have constant flag curvature [Bao et al. 2004], the Randers metric with navigation data $\left(Q_{k}, \tilde{X}\right)$, where

$$
X=\left(\begin{array}{cc}
d \sqrt{-1} & 0 \\
0 & -\frac{1}{n-1} d \sqrt{-1}
\end{array}\right), \quad \text { with }|d|<\sqrt{k},
$$

has constant flag curvature $k$. By the transformation formulas between the defining data and navigation data of Randers metrics [Chern and Shen 2005], we see that the Randers metrics with parameters as described in the theorem have constant flag curvature $k$.

Now we prove the converse of the conclusion above. Suppose $(M, F)$ is a connected simply connected homogeneous non-Riemannian Randers metric with constant positive flag curvature $k$. Suppose the underlying Riemannian metric is $Q$ and the corresponding vector field is $\tilde{X}$. Then $(M, Q)$ is a connected simply connected Riemannian metric with constant positive sectional curvature $k$. Thus $M$ is diffeomorphic to a sphere and $\tilde{X}$ is invariant under the full group of isometries of $(M, Q)$. In particular, $\tilde{X}$ has constant length with respect to $Q$. This means that the Randers metric is in the corrected Yasuda-Shimada family. That is, it satisfies $\theta=0$, and up to isometry there is only one family of such Randers metrics on odd-dimensional spheres [Bao et al. 2004]. Therefore they must be exactly the metrics constructed above.

Finally, by our previous result [An and Deng 2008], the Randers metric $F_{k, X}$ is of Douglas type if and only if $Q_{k}\left(X,[U, V]_{\mathfrak{m}}\right)=0$ for all $U, V \in \mathfrak{m}$. A simple direct computation shows that this holds only if $X=0$. Thus any non-Riemannian Randers metric constructed above is not of the Douglas type.

Other cases can be treated similarly; we omit the details here. The conclusion is that on each of the homogeneous manifolds other than the types VII, VIII, and IX, there exist invariant non-Riemannian Randers metrics. Any such metric is not of the Douglas type. On $\operatorname{Sp}(n) / \operatorname{Sp}(n-1)$ with $n \geq 2$, and given any $k>0$, there is also a family of invariant Randers metrics with constant flag curvature $k$. By the arguments above, this family must be isometric to the corresponding family on $\mathrm{SU}(2 n) / \mathrm{SU}(2 n-1)=S^{4 n-1}$ that has constant flag curvature $k$. Moreover, on $\operatorname{Spin}(9) / \operatorname{Spin}(7)$, since the isotropy representation has no fixed nonzero points [Ziller 1996], there are no Spin(9)-invariant non-Riemannian Randers metrics, although there do exist invariant non-Riemannian metrics. Of course, there are no invariant non-Riemannian metrics on the homogeneous manifolds of types VIII and IX.

\section{References}

[An and Deng 2008] H. An and S. Deng, "Invariant $(\alpha, \beta)$-metrics on homogeneous manifolds", Monatsh. Math. 154:2 (2008), 89-102. MR 2009b:53077 Zbl 05553530 
[Bao et al. 2000] D. Bao, S.-S. Chern, and Z. Shen, An introduction to Riemann-Finsler geometry, Graduate Texts in Mathematics 200, Springer, New York, 2000. MR 2001g:53130 Zbl 0954.53001

[Bao et al. 2004] D. Bao, C. Robles, and Z. Shen, "Zermelo navigation on Riemannian manifolds", J. Differential Geom. 66:3 (2004), 377-435. MR 2005k:58023 Zbl 1078.53073

[Berndt et al. 1997] J. Berndt, O. Kowalski, and L. Vanhecke, "Geodesics in weakly symmetric spaces", Ann. Global Anal. Geom. 15:2 (1997), 153-156. MR 98f:53037 Zbl 0880.53044

[Besse 1987] A. L. Besse, Einstein manifolds, Ergebnisse der Mathematik und ihrer Grenzgebiete

(3) 10, Springer, Berlin, 1987. MR 88f:53087 Zbl 0613.53001

[Chern and Shen 2005] S.-S. Chern and Z. Shen, Riemann-Finsler geometry, Nankai Tracts in Mathematics 6, World Scientific, Hackensack, NJ, 2005. MR 2006d:53094 Zbl 1085.53066

[Dadok 1982] J. Dadok, "On the $C^{\infty}$ Chevalley's theorem", Adv. in Math. 44:2 (1982), 121-131. MR 83m:53073 Zbl 0521.22009

[Dadok 1985] J. Dadok, "Polar coordinates induced by actions of compact Lie groups", Trans. Amer. Math. Soc. 288:1 (1985), 125-137. MR 86k:22019 Zbl 0565.22010

[D'Atri and Ziller 1979] J. E. D'Atri and W. Ziller, Naturally reductive metrics and Einstein metrics on compact Lie groups, Mem. Amer. Math. Soc. 18:215, Amer. Math. Soc., Providence, RI, 1979. MR 80i:53023 Zbl 0404.53044

[Deng and Hou 2002] S. Deng and Z. Hou, "The group of isometries of a Finsler space", Pacific J. Math. 207:1 (2002), 149-155. MR 2003m:53127 Zbl 1055.53055

[Deng and Hou 2004a] S. Deng and Z. Hou, "Invariant Finsler metrics on homogeneous manifolds", J. Phys. A 37:34 (2004), 8245-8253. MR 2005e:53112 Zbl 1062.58007

[Deng and Hou 2004b] S. Deng and Z. Hou, "Invariant Randers metrics on homogeneous Riemannian manifolds", J. Phys. A 37:15 (2004), 4353-4360. Corrected in 39:18 (2006), 5249-5250. MR 2005f:53125 Zbl 1049.83005

[Deng and Hou 2005a] S. Deng and Z. Hou, "Minkowski symmetric Lie algebras and symmetric Berwald spaces”, Geom. Dedicata 113 (2005), 95-105. MR 2006e:53131 Zbl 1084.53059

[Deng and Hou 2005b] S. Deng and Z. Hou, "On locally and globally symmetric Berwald spaces", J. Phys. A 38:8 (2005), 1691-1697. MR 2005j:53028

[Deng and Hou 2007] S. Deng and Z. Hou, "On symmetric Finsler spaces", Israel J. Math. 162 (2007), 197-219. MR 2008k:53161 Zbl 1141.53071

[Deng and Hou $\geq 2010$ ] S. Deng and Z. Hou, "Weakly symmetric Finsler spaces", preprint. To appear in Comm. Contemp. Math.

[Hano 1955] J.-i. Hano, "On affine transformations of a Riemannian manifold", Nagoya Math. J. 9 (1955), 99-109. MR 17,891d Zbl 0067.14601

[Helgason 1978] S. Helgason, Differential geometry, Lie groups, and symmetric spaces, Pure and Applied Mathematics 80, Academic Press, New York, 1978. MR 80k:53081 Zbl 0451.53038

[Kobayashi and Nomizu 1963] S. Kobayashi and K. Nomizu, Foundations of differential geometry, vol. 1, Wiley, New York, 1963. MR 27 \#2945 Zbl 0119.37502

[Kobayashi and Nomizu 1969] S. Kobayashi and K. Nomizu, Foundations of differential geometry, vol. 2, Interscience Tracts in Pure and Applied Mathematics 15, Wiley, New York, 1969. MR 38 \#6501 Zbl 0175.48504

[Kollross and Podestà 2003] A. Kollross and F. Podestà, "Homogeneous spaces with polar isotropy", Manuscripta Math. 110:4 (2003), 487-503. MR 2004b:53074 Zbl 1048.53035

[Korányi and Wolf 1965] A. Korányi and J. A. Wolf, "Realization of hermitian symmetric spaces as generalized half-planes”, Ann. of Math. (2) 81 (1965), 265-288. MR 30 \#4980 Zbl 0137.27402

[Kostant 1973] B. Kostant, "On convexity, the Weyl group and the Iwasawa decomposition", Ann. Sci. École Norm. Sup. (4) 6 (1973), 413-455. MR 51 \#806 Zbl 0293.22019 
[Krämer 1975] M. Krämer, "Eine Klassifikation bestimmter Untergruppen kompakter zusammenhängender Liegruppen”, Comm. Algebra 3:8 (1975), 691-737. MR 51 \#13140 Zbl 0309.22013

[Manturov 1961a] O. V. Manturov, "Homogeneous, non-symmetric Riemannian spaces with an irreducible rotation group", Dokl. Akad. Nauk SSSR 141 (1961), 792-795. In Russian; translated in Sov. Math., Dokl. 2 (1961), 1550-1554. MR 25 \#2552 Zbl Zbl 0188.26502

[Manturov 1961b] O. V. Manturov, "Riemannian spaces with orthogonal and symplectic motion groups and an irreducible rotation group", Dokl. Akad. Nauk SSSR 141 (1961), 1034-1037. In Russian; translated in Sov. Math., Dokl 2 (1961), 1034-1037. MR 25 \#2551

[Manturov 1966] O. V. Manturov, "Homogeneous Riemannian spaces with an irreducible rotation group”, Trudy Sem. Vektor. Tenzor. Anal. 13 (1966), 68-145. In Russian. MR 35 \#926 Zbl 0173. 24102

[Nguyen 2000] H. D. Nguyêñ, "Compact weakly symmetric spaces and spherical pairs", Proc. Amer. Math. Soc. 128:11 (2000), 3425-3433. MR 2001b:53060 Zbl 0976.53056

[Oniščik 1963] A. L. Oniščik, "Transitive compact transformation groups", Mat. Sb. (N.S.) 60 (102) (1963), 447-485. In Russian; translated in Amer. Math. Soc. Trans., 55:2 (1966), 153-194. MR 27 \#5868

[Shen 2009] Z. Shen, "Some open problems in Finsler geometry", preprint, 2009, available at www.math.iupui.edu/ zshen/Research/papers/Problem.pdf.

[Szabó 1981] Z. I. Szabó, "Positive definite Berwald spaces: Structure theorems on Berwald spaces", Tensor (N.S.) 35:1 (1981), 25-39. MR 82f:53043 Zbl 0464.53025

[Szabó 2006] Z. I. Szabó, "Berwald metrics constructed by Chevalley polynomials", preprint, 2006. arXiv math/0601522

[Tam 1998] T.-Y. Tam, “A unified extension of two results of Ky Fan on the sum of matrices", Proc. Amer. Math. Soc. 126:9 (1998), 2607-2614. MR 98m:15032 Zbl 0912.15018

[Wang and Ziller 1991] M. Wang and W. Ziller, "On isotropy irreducible Riemannian manifolds", Acta Math. 166:3-4 (1991), 223-261. MR 92b:53078 Zbl 0732.53040

[Wolf 1968] J. A. Wolf, "The goemetry and structure of isotropy irreducible homogeneous spaces", Acta Math. 120 (1968), 59-148. Correction in 152 (1984), 141-142. MR 36 \#6549 Zbl 0157.52102

[Wolf 1977] J. A. Wolf, Spaces of constant curvature, 4th ed., Publish or Perish, Houston, 1977. MR 88k:53002 Zbl 0281.53034

[Yakimova 2004] O. S. Yakimova, "Weakly symmetric Riemannian manifolds with a reductive isometry group", Mat. Sb. 195:4 (2004), 143-160. In Russian; translated in Sb. Math. 195:3-4 (2004), 143-160. MR 2005f:53081 Zbl 1078.53043

[Ziller 1996] W. Ziller, "Weakly symmetric spaces", pp. 355-368 in Topics in geometry, edited by S. Gindikin, Progr. Nonlinear Differential Equations Appl. 20, Birkhäuser, Boston, MA, 1996. MR 97c:53081 Zbl 0860.53030

Received April 30, 2009.

\section{SHAOQIANG DENG}

SCHOOL OF MATHEMATICAL SCIENCES AND LPMC

NANKAI UNIVERSITY

TIANJIN 300071

CHINA

dengsq@nankai.edu.cn 


\title{
PACIFIC JOURNAL OF MATHEMATICS
}

\author{
http://www.pjmath.org \\ Founded in 1951 by \\ E. F. Beckenbach (1906-1982) and F. Wolf (1904-1989)
}

\section{EDITORS}

V. S. Varadarajan (Managing Editor)

Department of Mathematics

University of California

Los Angeles, CA 90095-1555

pacific@math.ucla.edu

Vyjayanthi Chari

Department of Mathematics

University of California

Riverside, CA 92521-0135

chari@math.ucr.edu

Robert Finn

Department of Mathematics Stanford University

Stanford, CA 94305-2125

finn@math.stanford.edu

Kefeng Liu

Department of Mathematics

University of California

Los Angeles, CA 90095-1555

liu@math.ucla.edu
Darren Long

Department of Mathematics

University of California

Santa Barbara, CA 93106-3080

long@math.ucsb.edu

Jiang-Hua Lu

Department of Mathematics

The University of Hong Kong

Pokfulam Rd., Hong Kong jhlu@maths.hku.hk

Alexander Merkurjev

Department of Mathematics

University of California

Los Angeles, CA 90095-1555

merkurev@math.ucla.edu
Sorin Popa

Department of Mathematics University of California

Los Angeles, CA 90095-1555 popa@math.ucla.edu

Jie Qing

Department of Mathematics

University of California

Santa Cruz, CA 95064

qing@cats.ucsc.edu

Jonathan Rogawski

Department of Mathematics

University of California

Los Angeles, CA 90095-1555

jonr@math.ucla.edu

\section{PRODUCTION}

pacific@math.berkeley.edu

\begin{abstract}
Silvio Levy, Scientific Editor Matthew Cargo, Senior Production Editor
\end{abstract}
ACADEMIA SINICA, TAIPEI

CALIFORNIA INST. OF TECHNOLOGY

INST. DE MATEMÁTICA PURA E APLICADA

KEIO UNIVERSITY

MATH. SCIENCES RESEARCH INSTITUTE

NEW MEXICO STATE UNIV.

OREGON STATE UNIV.

\section{SUPPORTING INSTITUTIONS}

STANFORD UNIVERSITY
UNIV. OF BRITISH COLUMBIA
UNIV. OF CALIFORNIA, BERKELEY
UNIV. OF CALIFORNIA, DAVIS
UNIV. OF CALIFORNIA, LOS ANGELES
UNIV. OF CALIFORNIA, RIVERSIDE
UNIV. OF CALIFORNIA, SAN DIEGO
UNIV. OF CALIF., SANTA BARBARA

UNIV. OF CALIF., SANTA CRUZ

UNIV. OF MONTANA

UNIV. OF OREGON

UNIV. OF SOUTHERN CALIFORNIA

UNIV. OF UTAH

UNIV. OF WASHINGTON

WASHINGTON STATE UNIVERSITY

These supporting institutions contribute to the cost of publication of this Journal, but they are not owners or publishers and have no responsibility for its contents or policies.

See inside back cover or www.pjmath.org for submission instructions.

The subscription price for 2010 is US \$420/year for the electronic version, and \$485/year for print and electronic.

Subscriptions, requests for back issues from the last three years and changes of subscribers address should be sent to Pacific Journal of Mathematics, P.O. Box 4163, Berkeley, CA 94704-0163, U.S.A. Prior back issues are obtainable from Periodicals Service Company, 11 Main Street, Germantown, NY 12526-5635. The Pacific Journal of Mathematics is indexed by Mathematical Reviews, Zentralblatt MATH, PASCAL CNRS Index, Referativnyi Zhurnal, Current Mathematical Publications and the Science Citation Index.

The Pacific Journal of Mathematics (ISSN 0030-8730) at the University of California, c/o Department of Mathematics, 969 Evans Hall, Berkeley, CA 94720-3840, is published monthly except July and August. Periodical rate postage paid at Berkeley, CA 94704, and additional mailing offices. POSTMASTER: send address changes to Pacific Journal of Mathematics, P.O. Box 4163, Berkeley, CA 94704-0163.

PJM peer review and production are managed by EditFLOW ${ }^{\mathrm{TM}}$ from Mathematical Sciences Publishers.

PUBLISHED BY PACIFIC JOURNAL OF MATHEMATICS

at the University of California, Berkeley 94720-3840

A NON-PROFIT CORPORATION

Typeset in LATEX

Copyright $(\mathrm{C} 2010$ by Pacific Journal of Mathematics 


\section{PACIFIC JOURNAL OF MATHEMATICS}

Volume $247 \quad$ No. $1 \quad$ September 2010

Classification results for easy quantum groups

TEOdor BANiCA, StePhen CURRAN and Roland SPEICHER

Batalin-Vilkovisky coalgebra of string topology

XiAOJUn CHEN and WeE LiAnG GAN

Invariant Finsler metrics on polar homogeneous spaces

SHAOQIANG DENG

A proof of the Concus-Finn conjecture

KIRK E. LANCASTER

The existence and monotonicity of a three-dimensional transonic shock in a finite nozzle with axisymmetric exit pressure

JUn Li, ZHOUPING XIN and HUICHENG YiN

Bi-Hamiltonian flows and their realizations as curves in real semisimple homogeneous manifolds

GLORIA MARÍ BEFFA

Closed orbits of a charge in a weakly exact magnetic field

WILL J. MERRY

Ringel-Hall algebras and two-parameter quantized enveloping algebras

XIN TANG

A new probability distribution with applications

MINGJIN WANG 